Acta Crystallographica Section E

Structure Reports

Online

ISSN 1600-5368

\section{Gibberellin A4 monohydrate}

\section{Yun-Fen Hua, Hai Yan Li, Lin Lin Ma, Xing Zhang and Zhi Min Jin*}

College of Pharmaceutical Sciences, Zhejiang University of Technology, Hangzhou 310014, People's Republic of China

Correspondence e-mail: apharm@sina.com

Received 9 November 2007; accepted 19 November 2007

Key indicators: single-crystal X-ray study; $T=193 \mathrm{~K}$; mean $\sigma(\mathrm{C}-\mathrm{C})=0.011 \AA$;

$R$ factor $=0.077 ; w R$ factor $=0.207 ;$ data-to-parameter ratio $=6.9$.

The title compond, $\mathrm{C}_{19} \mathrm{H}_{24} \mathrm{O}_{5} \cdot \mathrm{H}_{2} \mathrm{O}$, has two gibberellin $\mathrm{A} 4$ molecules and two water molecules in the asymmetric unit. The $A$ and $B$ rings have chair conformations, whereas the $C$ and $D$ rings have envelope conformations; the two rings which contain the lactone and carbonyl bridge adopt chair and envelope conformations. The crystal structure is established by $\mathrm{O}-\mathrm{H} \cdots \mathrm{O}$ hydrogen bonds and supported by $\mathrm{C}-\mathrm{H} \cdots \mathrm{O}$ hydrogen bonds.

\section{Related literature}

For related literature, see: Coggins et al. (1969); Ellames et al. (1979); Hossain et al. (1988); Komoda et al. (1968); Kutschabsky \& Adam (1983); Thompson et al. (2000); Furber et al. (1992); Nagata et al. (1971); Poling (1991).

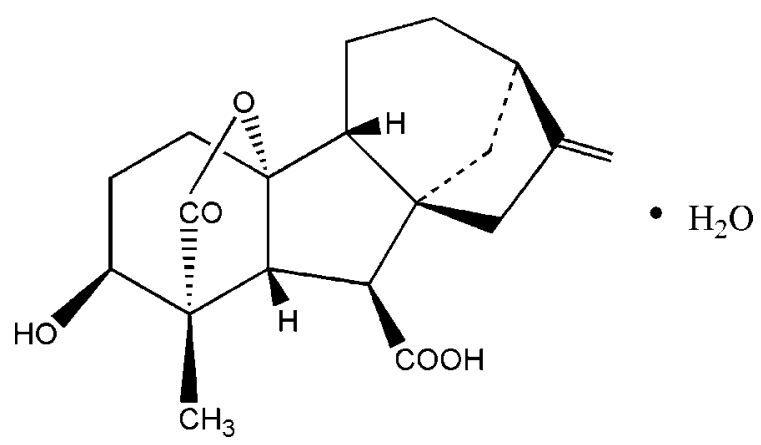

\section{Experimental}

\section{Crystal data}

$\mathrm{C}_{19} \mathrm{H}_{24} \mathrm{O}_{5} \cdot \mathrm{H}_{2} \mathrm{O}$

$M_{r}=350.40$

$a=9.6466(13) \AA$

$b=18.968(3) \AA$

$c=9.4910(12) \AA$

$\beta=89.989$ (3) ${ }^{\circ}$
Monoclinic, $P 2_{1}$
$V=1736.6(4) \AA^{3}$

$Z=4$

$\mu=0.10 \mathrm{~mm}^{-1}$

$T=193$ (2) K

$0.44 \times 0.35 \times 0.19 \mathrm{~mm}$
Mo $K \alpha$ radiation
Data collection

Rigaku Mercury diffractometer

Absorption correction: multi-scan (SHELXTL; Siemens, 1998)

$T_{\min }=0.958, T_{\max }=0.981$

17165 measured reflections 3080 reflections with $I>2 \sigma(I)$ $R_{\text {int }}=0.047$

Refinement

$R\left[F^{2}>2 \sigma\left(F^{2}\right)\right]=0.077$

$w R\left(F^{2}\right)=0.207$

$S=1.13$

475 parameters

7 restraints

$\mathrm{H}$ atoms treated by a mixture of independent and constrained refinement

$\Delta \rho_{\max }=0.38{\mathrm{e} \AA^{-3}}^{-3}$

$\Delta \rho_{\min }=-0.38$ e $\AA^{-3}$ 3281 independent reflections

3281 reflections

Table 1

Hydrogen-bond geometry $\left(\AA{ }^{\circ}\right)$.

\begin{tabular}{|c|c|c|c|c|}
\hline$D-\mathrm{H} \cdots A$ & $D-\mathrm{H}$ & $\mathrm{H} \cdots A$ & $D \cdots A$ & $D-\mathrm{H} \cdots A$ \\
\hline $\mathrm{O} 3-\mathrm{H} 3 \cdots \mathrm{O} 11^{\mathrm{i}}$ & 0.84 & 2.01 & $2.825(8)$ & $163(4)$ \\
\hline $\mathrm{O} 4-\mathrm{H} 4 \cdots \mathrm{O}^{\mathrm{ii}}$ & 0.84 & 1.83 & $2.634(8)$ & $158(6)$ \\
\hline $\mathrm{O} 6-\mathrm{H} 6 W A \cdots{ }^{\mathrm{iii}}$ & $0.82(5)$ & $2.15(6)$ & $2.939(9)$ & $161(6)$ \\
\hline $\mathrm{O} 6-\mathrm{H} 6 W B \cdots \mathrm{O} 4^{\mathrm{iv}}$ & $0.82(5)$ & $1.93(13)$ & $2.634(9)$ & $143(6)$ \\
\hline $\mathrm{O} 10-\mathrm{H} 10 \cdots \mathrm{O} 12$ & $0.83(7)$ & $1.81(6)$ & $2.625(8)$ & $163(4)$ \\
\hline $\mathrm{O} 12-\mathrm{H} 12 A \cdots \mathrm{O} 2$ & $0.82(9)$ & $2.16(11)$ & $2.925(10)$ & $154(10)$ \\
\hline $\mathrm{O} 12-\mathrm{H} 12 B \cdots \mathrm{O} 9^{\mathrm{iv}}$ & $0.82(9)$ & $1.91(6)$ & $2.727(10)$ & $167(2)$ \\
\hline $\mathrm{C} 11-\mathrm{H} 11 \cdots \mathrm{O} 8$ & 1.00 & 2.54 & $3.531(10)$ & $173(7)$ \\
\hline $\mathrm{C} 30-\mathrm{H} 30 \cdots \mathrm{O} 2^{v}$ & 1.00 & 2.46 & $3.442(9)$ & $166(7)$ \\
\hline
\end{tabular}

Symmetry codes: (i) $-x+1, y+\frac{1}{2},-z+1$; (ii) $x, y, z+1$; (iii) $x-1, y, z-1$; (iv) $x, y, z-1 ;(\mathrm{v}) x+1, y, z$

Data collection: PROCESS-AUTO (Rigaku, 1998); cell refinement: PROCESS-AUTO; data reduction: CrystalStructure (Rigaku/ MSC, 2004); program(s) used to solve structure: SHELXS97 (Sheldrick, 1997); program(s) used to refine structure: SHELXL97 (Sheldrick, 1997); molecular graphics: SHELXTL (Siemens, 1998); software used to prepare material for publication: SHELXTL.

Supplementary data and figures for this paper are available from the IUCr electronic archives (Reference: AT2480).

\section{References}

Coggins, C. W., Scora, R. W., Lewis, L. N. \& Knapp, J. C. F. (1969). J. Agric. Food Chem. 17, 805-806.

Ellames, G., Hanson, J. R., Hitchcock, P. B. \& Thomas, S. A. (1979). J. Chem. Soc. Perkin Trans. 1, pp. 1922-1926.

Furber, M., Mander, L. N., Patrick, G. L. \& Willis, A. C. (1992). Acta Cryst. C48, 1348-1350.

Hossain, M. B., van der Helm, D., Sanduja, R. \& Alam, M. (1988). Acta Cryst. C44, 1022-1024.

Komoda, Y., Isogai, Y. \& Okamoto, T. (1968). Sci. Pap. Coll. Gen. Educ. Univ. Tokyo, 18, 221-223.

Kutschabsky, L. \& Adam, G. (1983). J. Chem. Soc. Perkin Trans. 1, pp. 1653 1655 .

Nagata, W., Wakabayashi, T., Narisada, M., Hayase, Y. \& Kamata, S. (1971). J. Am. Chem. Soc. 93, 5740-5758.

Poling, S. M. (1991). J. Agric. Food Chem. 39, 677-680.

Rigaku (1998). PROCESS-AUTO. Rigaku Corporation, Tokyo, Japan.

Rigaku/MSC (2004). CrystalStructure. Version 3.6.0. Rigaku/MSC, The Woodlands, Texas, USA.

Sheldrick, G. M. (1997). SHELXS97 and SHELXL97. University of Göttingen, Germany.

Siemens (1998). SHELXTL. Siemens Analytical X-ray Instruments Inc., Madison, Wisconsin, USA.

Thompson, H. W., Brunskill, A. P. J. \& Lalancette, R. A. (2000). Acta Cryst. C56, 1507-1509. 


\section{supporting information}

Acta Cryst. (2008). E64, o30 [https://doi.org/10.1107/S160053680706062X]

\section{Gibberellin A4 monohydrate}

\section{Yun-Fen Hua, Hai Yan Li, Lin Lin Ma, Xing Zhang and Zhi Min Jin}

\section{S1. Comment}

The gibberellins are an important family of diterpenoid plant-growth factors. It is used mainly to improve the rind quality of fruit (Coggins et al., 1969; Poling, 1991). Some crystal sructures of gibberellins are reported previously, such as gibberellin A20 (Komoda et al., 1968), gibberellin A4 trmethyl ester (Ellames et al., 1979), gibberellin A3 (Kutschabsky \& Adam, 1983), 3-O-acetylgiberellin A3 (Hossain et al., 1988), gibberellin C (Thompson et al., 2000), 15crclogibberellin A9 (Furber et al., 1992), and gibberellin A15 (Nagata et al., 1971). We present here the structure of the title compound, (I).

As shown in Fig. 1, there are two crystallographically independent (I) in the asymmeytric unit cell. The $\mathrm{H}$ atom at $\mathrm{C} 2$ and $\mathrm{C} 8$, the methylat $\mathrm{C} 3$, the hdroxyl at $\mathrm{C} 5$, the methano $(\mathrm{C} 15)$ bridge and the carboxyl, all lie on the 'upper' $\beta$ face of the molecule, and the $\mathrm{C}=\mathrm{O}$ group also toward the $\beta$ face. Only the bridge that links the lactone and carbonyl has $\alpha$ stereochemistry. The A ring and the $\mathrm{B}$ ring have chair conformations, whereas the $\mathrm{C}$ ring and $\mathrm{D}$ ring have the envelope conformations, besides those, the two rings which contain the lactone and carbonyl bridge adopt chair and envelope conformations respectively. The $\mathrm{H}$ atom of axial hydroxyl group (O10) is aimed away from the ring system toward a water molecule, and the water (O12) hydrogen-bonding relates to the lactone carbonyl (O2). There exists two counterdirectional screw-related sets of helices, the hydroxyl and lactone carbonyl in related molecules within a given helix are involved in hydrogen bonds from a single water of hydration (O12- $\mathrm{H} 12 \mathrm{~A} \cdots \mathrm{O} 2$ and $\mathrm{O} 12-\mathrm{H} 12 \mathrm{~B} \cdots \mathrm{O} 9)$, in turn, the oxygen (O12) of the same water accepts a hydrogen bond from the carboxyl group of a third screw-related molecule in an adjacent counter-directionally oriented helix $(\mathrm{O} 10-\mathrm{H} 10 \cdots \mathrm{O} 12)$. Thus, water molecule acts both to brace the helix and to bridge it, alternately, to two different screw-related neighboring chains in a complex three-dimensional array.

The water molecules are linked to the GA4 molecules by $\mathrm{O}-\mathrm{H} \cdots \mathrm{O}$ hydrogen bonds (Table 2), more over, weak $\mathrm{C}-$ $\mathrm{H} \cdots \mathrm{O}$ hydrogen bonds are obeserved between the gibberellin molecules to help increasing the stalitiy of the crystal (Fig. 2 $\&$ Table 2).

\section{S2. Experimental}

Gibberellin A4 (1 mmol, $0.33 \mathrm{~g})$ was dissolved with $10 \mathrm{ml}$ tetrahydrofuran and water (1:1) admixture, then heated to boiling and stirred for ten minutes. The resulting solution was cooled to the room temperature and the colorless crystals were collected after five days.

\section{S3. Refinement}

The $\mathrm{H}$ atoms attaching to $\mathrm{O}$ atoms and the $\mathrm{H}$ atoms of water were deduced from difference Fourier maps, and incorporated in refinement. Others were placed in calculated positions and allowed to ride on their parent atoms at distances of 0.98 (methyl), 0.99 (methylene), $1.00 \AA$ (methine), with $U_{\text {iso }}(\mathrm{H})$ values 1.2 times $U_{\text {eq }}$ of the parent atoms. Because of the lack of atoms heavier than oxygen and the short measuring wavelength of Mo radiation, no useful 
absolute structure parameter could be refined.

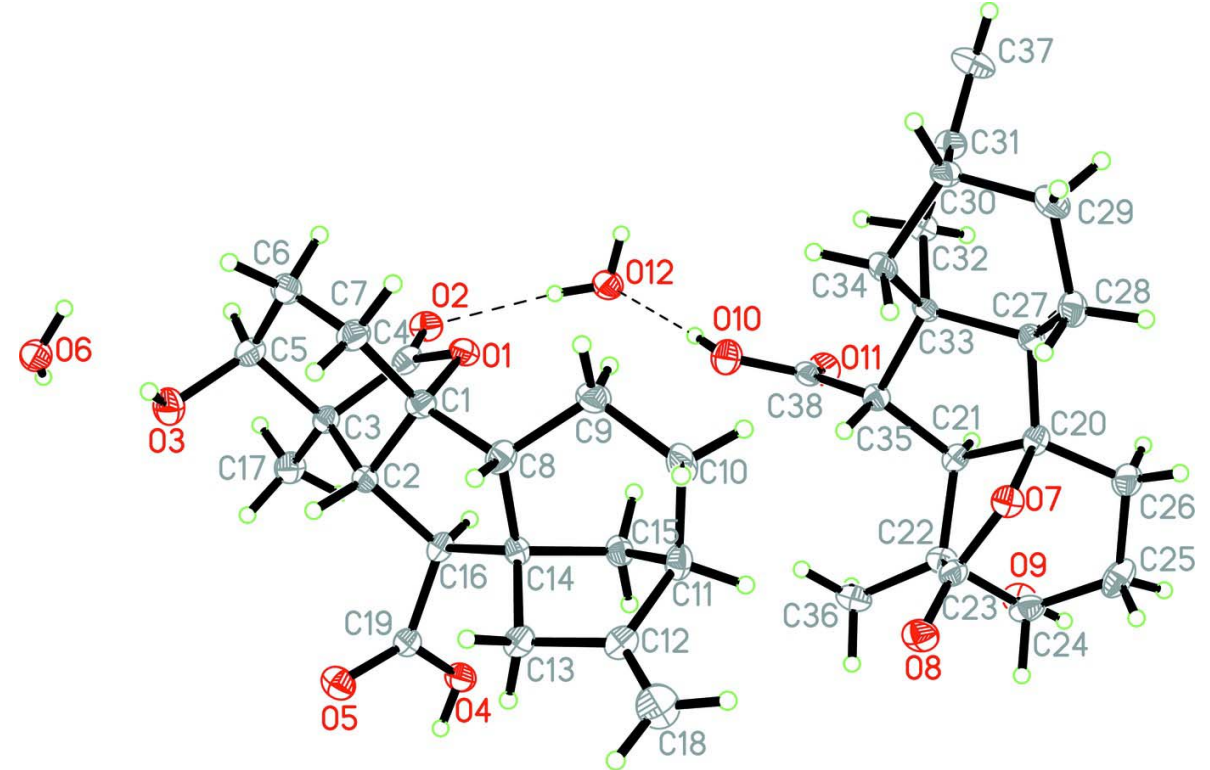

Figure 1

The cell unit of (I) with atom labels, showing $40 \%$ probability displacement ellipsoids. The thin lines denote the hydrogen bonds. 


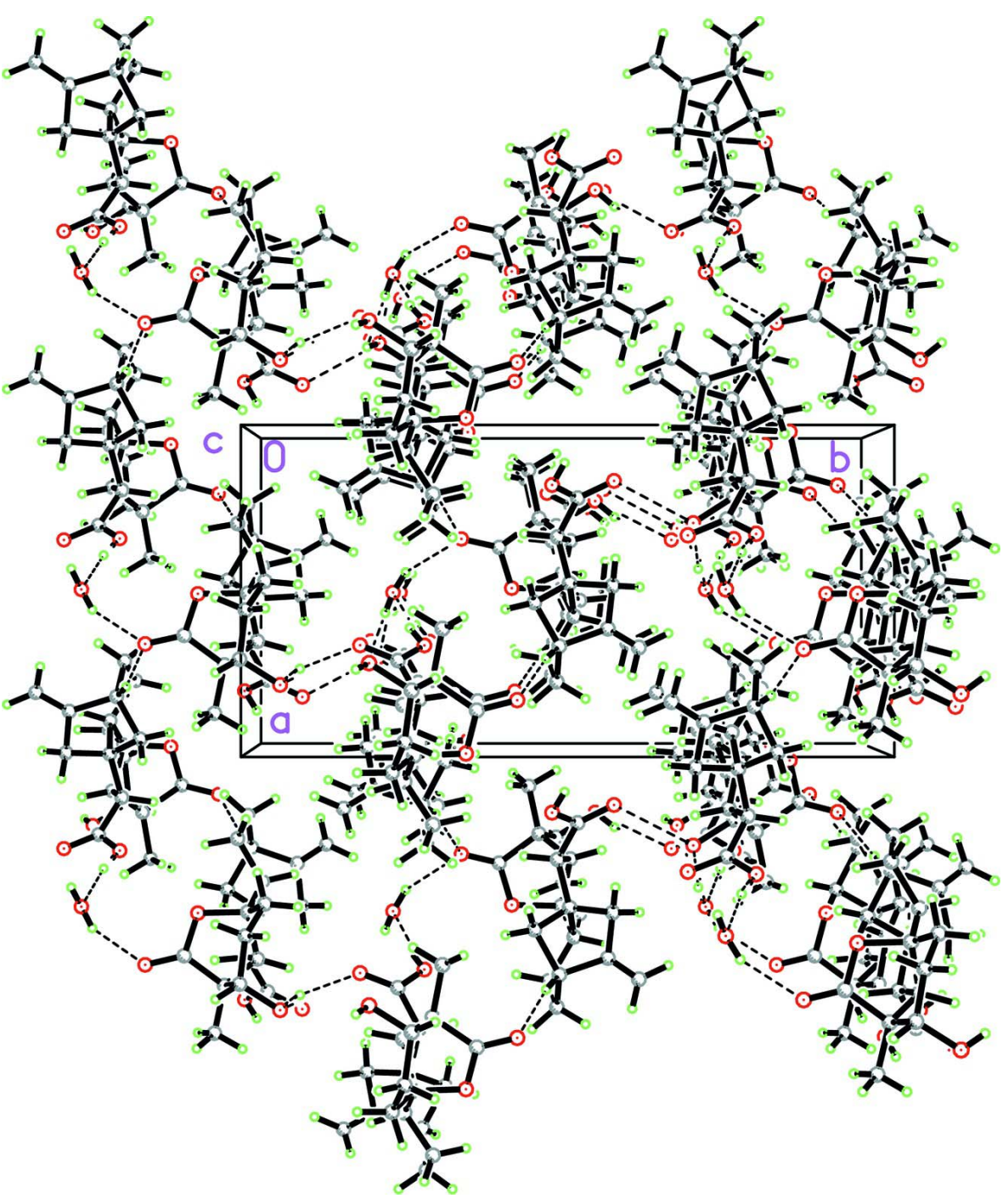

Figure 2

The crystal packing of (I) viewed down along the $c$ axis, hydrogen bonds shown as thin lines.

Gibberellin A4 monohydrate

Crystal data

$\mathrm{C}_{19} \mathrm{H}_{24} \mathrm{O}_{5} \cdot \mathrm{H}_{2} \mathrm{O}$

$M_{r}=350.40$

Monoclinic, $P 2_{1}$

Hall symbol: P 2yb

$a=9.6466$ (13) $\AA$

$b=18.968(3) \AA$

$c=9.4910$ (12) $\AA$

$\beta=89.989(3)^{\circ}$

$V=1736.6(4) \AA^{3}$

$Z=4$
$F(000)=752$

$D_{\mathrm{x}}=1.340 \mathrm{Mg} \mathrm{m}^{-3}$

Mo $K \alpha$ radiation, $\lambda=0.71070 \AA$

Cell parameters from 6798 reflections

$\theta=3.0-25.3^{\circ}$

$\mu=0.10 \mathrm{~mm}^{-1}$

$T=193 \mathrm{~K}$

Prism, colourless

$0.44 \times 0.35 \times 0.19 \mathrm{~mm}$ 


\section{Data collection}

Rigaku Mercury diffractometer

Radiation source: fine-focus sealed tube Graphite monochromator $\omega$ scans

Absorption correction: multi-scan (SHELXTL; Siemens, 1998)

$T_{\text {min }}=0.958, T_{\max }=0.981$

\section{Refinement}

Refinement on $F^{2}$

Least-squares matrix: full

$R\left[F^{2}>2 \sigma\left(F^{2}\right)\right]=0.077$

$w R\left(F^{2}\right)=0.207$

$S=1.13$

3281 reflections

475 parameters

7 restraints

Primary atom site location: structure-invariant direct methods

Secondary atom site location: difference Fourier map
17165 measured reflections

3281 independent reflections

3080 reflections with $I>2 \sigma(I)$

$R_{\text {int }}=0.047$

$\theta_{\max }=25.4^{\circ}, \theta_{\min }=3.0^{\circ}$

$h=-11 \rightarrow 11$

$k=-20 \rightarrow 22$

$l=-11 \rightarrow 11$

Hydrogen site location: inferred from neighbouring sites

$\mathrm{H}$ atoms treated by a mixture of independent and constrained refinement

$w=1 /\left[\sigma^{2}\left(F_{\mathrm{o}}^{2}\right)+(0.0724 P)^{2}+5.3874 P\right]$ where $P=\left(F_{\mathrm{o}}{ }^{2}+2 F_{\mathrm{c}}{ }^{2}\right) / 3$

$(\Delta / \sigma)_{\max }<0.001$

$\Delta \rho_{\max }=0.38$ e $\AA^{-3}$

$\Delta \rho_{\min }=-0.38$ e $\AA^{-3}$

Extinction correction: SHELXL97 (Sheldrick, 1997), $\mathrm{Fc}^{*}=\mathrm{kFc}\left[1+0.001 \times \mathrm{xF}^{2} \lambda^{3} / \sin (2 \theta)\right]^{-1 / 4}$

Extinction coefficient: $0.030(4)$

\section{Special details}

Geometry. All e.s.d.'s (except the e.s.d. in the dihedral angle between two 1.s. planes) are estimated using the full covariance matrix. The cell e.s.d.'s are taken into account individually in the estimation of e.s.d.'s in distances, angles and torsion angles; correlations between e.s.d.'s in cell parameters are only used when they are defined by crystal symmetry. An approximate (isotropic) treatment of cell e.s.d.'s is used for estimating e.s.d.'s involving 1.s. planes.

Refinement. Refinement of $F^{2}$ against ALL reflections. The weighted $R$-factor $w R$ and goodness of fit $S$ are based on $F^{2}$, conventional $R$-factors $R$ are based on $F$, with $F$ set to zero for negative $F^{2}$. The threshold expression of $F^{2}>\sigma\left(F^{2}\right)$ is used only for calculating $R$-factors(gt) etc. and is not relevant to the choice of reflections for refinement. $R$-factors based on $F^{2}$ are statistically about twice as large as those based on $F$, and $R$ - factors based on ALL data will be even larger.

Fractional atomic coordinates and isotropic or equivalent isotropic displacement parameters $\left(\AA^{2}\right)$

\begin{tabular}{lllll}
\hline & $x$ & $y$ & $z$ & $U_{\text {iso }}^{*} / U_{\text {eq }}$ \\
\hline O1 & $0.4911(5)$ & $0.4162(3)$ & $0.4548(5)$ & $0.0302(12)$ \\
O2 & $0.3349(6)$ & $0.3352(3)$ & $0.3931(6)$ & $0.0342(13)$ \\
O3 & $0.2111(6)$ & $0.5532(3)$ & $0.2383(7)$ & $0.0401(14)$ \\
H3 & 0.2615 & 0.5851 & 0.2043 & $0.060^{*}$ \\
O4 & $0.1765(6)$ & $0.4775(3)$ & $0.8495(6)$ & $0.0346(13)$ \\
H4 & 0.1107 & 0.4964 & 0.8936 & $0.052^{*}$ \\
O5 & $0.1604(6)$ & $0.5757(3)$ & $0.7208(6)$ & $0.0376(13)$ \\
O6 & $0.0054(6)$ & $0.5236(3)$ & $0.0452(6)$ & $0.0377(14)$ \\
O7 & $0.9843(5)$ & $0.3447(3)$ & $1.1202(6)$ & $0.0308(12)$ \\
O8 & $0.8233(6)$ & $0.4220(3)$ & $1.1879(6)$ & $0.0385(14)$ \\
O9 & $0.7109(6)$ & $0.2016(3)$ & $1.3376(6)$ & $0.0392(14)$ \\
H9 & 0.7274 & 0.1835 & 1.4166 & $0.059^{*}$ \\
O10 & $0.6679(6)$ & $0.2784(3)$ & $0.7237(6)$ & $0.0396(14)$ \\
H10 & 0.6143 & 0.2548 & 0.6722 & $0.059^{*}$ \\
O11 & $0.6654(6)$ & $0.1799(3)$ & $0.8549(6)$ & $0.0371(14)$
\end{tabular}




\begin{tabular}{|c|c|c|c|c|}
\hline $\mathrm{O} 12$ & $0.5048(6)$ & $0.2267(4)$ & $0.5279(6)$ & $0.0382(14)$ \\
\hline $\mathrm{C} 1$ & $0.4931(8)$ & 0.4951 (4) & $0.4695(8)$ & 0.0287 (17) \\
\hline $\mathrm{C} 2$ & $0.3418(7)$ & $0.5094(4)$ & $0.5070(7)$ & $0.0256(16)$ \\
\hline $\mathrm{H} 2$ & 0.3155 & 0.5599 & 0.4920 & $0.031 *$ \\
\hline $\mathrm{C} 3$ & $0.2695(8)$ & $0.4602(4)$ & $0.4041(8)$ & $0.0259(16)$ \\
\hline $\mathrm{C} 4$ & $0.3615(8)$ & $0.3961(4)$ & $0.4122(8)$ & $0.0304(17)$ \\
\hline $\mathrm{C} 5$ & $0.2899(9)$ & $0.4894(5)$ & $0.2509(8)$ & $0.0359(19)$ \\
\hline H5 & 0.2502 & 0.4542 & 0.1837 & $0.043 *$ \\
\hline C6 & $0.4416(9)$ & $0.4998(5)$ & $0.2134(9)$ & 0.039 (2) \\
\hline H6A & 0.4797 & 0.4541 & 0.1813 & $0.046^{*}$ \\
\hline H6B & 0.4472 & 0.5331 & 0.1332 & $0.046^{*}$ \\
\hline C7 & $0.5301(9)$ & $0.5267(5)$ & $0.3279(8)$ & $0.0359(19)$ \\
\hline H7A & 0.5206 & 0.5786 & 0.3332 & $0.043^{*}$ \\
\hline H7B & 0.6282 & 0.5158 & 0.3062 & $0.043^{*}$ \\
\hline $\mathrm{C} 8$ & $0.5786(8)$ & $0.5146(5)$ & $0.5987(8)$ & $0.0329(18)$ \\
\hline H8 & 0.6024 & 0.5658 & 0.5910 & $0.039^{*}$ \\
\hline $\mathrm{C} 9$ & $0.7138(8)$ & $0.4740(5)$ & $0.6141(9)$ & $0.039(2)$ \\
\hline H9A & 0.7798 & 0.4897 & 0.5406 & $0.047 *$ \\
\hline H9B & 0.6960 & 0.4231 & 0.5999 & $0.047^{*}$ \\
\hline $\mathrm{C} 10$ & $0.7792(9)$ & $0.4857(6)$ & $0.7620(10)$ & $0.044(2)$ \\
\hline $\mathrm{H} 10 \mathrm{~A}$ & 0.8449 & 0.4469 & 0.7823 & $0.052^{*}$ \\
\hline H10B & 0.8319 & 0.5305 & 0.7619 & $0.052^{*}$ \\
\hline C11 & $0.6678(8)$ & 0.4884 (4) & $0.8784(9)$ & $0.0328(18)$ \\
\hline H11 & 0.7037 & 0.4674 & 0.9678 & $0.039^{*}$ \\
\hline $\mathrm{C} 12$ & $0.6146(8)$ & $0.5632(4)$ & $0.9034(8)$ & $0.0325(18)$ \\
\hline $\mathrm{C} 13$ & $0.4837(8)$ & $0.5720(4)$ & $0.8192(8)$ & 0.0307 (17) \\
\hline H13A & 0.4881 & 0.6150 & 0.7603 & $0.037^{*}$ \\
\hline H13B & 0.4019 & 0.5751 & 0.8819 & $0.037^{*}$ \\
\hline C14 & $0.4764(8)$ & $0.5059(4)$ & $0.7271(8)$ & $0.0260(16)$ \\
\hline $\mathrm{C} 15$ & $0.5351(8)$ & $0.4502(5)$ & $0.8300(8)$ & $0.0316(17)$ \\
\hline H15A & 0.4712 & 0.4413 & 0.9097 & $0.038^{*}$ \\
\hline H15B & 0.5563 & 0.4053 & 0.7817 & $0.038^{*}$ \\
\hline $\mathrm{C} 16$ & $0.3326(7)$ & $0.4877(4)$ & $0.6617(7)$ & $0.0244(15)$ \\
\hline H16 & 0.3225 & 0.4353 & 0.6643 & $0.029^{*}$ \\
\hline $\mathrm{C} 17$ & $0.1175(8)$ & $0.4436(5)$ & $0.4304(9)$ & 0.0368 (19) \\
\hline H17A & 0.0794 & 0.4185 & 0.3488 & $0.055^{*}$ \\
\hline H17B & 0.0662 & 0.4876 & 0.4448 & $0.055^{*}$ \\
\hline $\mathrm{H} 17 \mathrm{C}$ & 0.1090 & 0.4139 & 0.5145 & $0.055^{*}$ \\
\hline C18 & $0.6703(11)$ & $0.6092(6)$ & $0.9877(12)$ & $0.054(3)$ \\
\hline H18A & 0.6275 & 0.6538 & 1.0009 & $0.064^{*}$ \\
\hline H18B & 0.7537 & 0.5982 & 1.0362 & $0.064^{*}$ \\
\hline C19 & $0.2140(8)$ & $0.5185(4)$ & $0.7452(8)$ & $0.0267(16)$ \\
\hline $\mathrm{C} 20$ & $0.9918(8)$ & $0.2658(4)$ & $1.0997(8)$ & $0.0274(16)$ \\
\hline $\mathrm{C} 21$ & $0.8411(8)$ & $0.2487(4)$ & $1.0648(7)$ & $0.0253(15)$ \\
\hline $\mathrm{H} 21$ & 0.8199 & 0.1974 & 1.0771 & $0.030^{*}$ \\
\hline $\mathrm{C} 22$ & $0.7644(8)$ & $0.2944(4)$ & $1.1708(8)$ & $0.0292(17)$ \\
\hline $\mathrm{C} 23$ & $0.8545(8)$ & $0.3612(4)$ & $1.1630(8)$ & $0.0292(17)$ \\
\hline C24 & $0.7910(10)$ & $0.2646(4)$ & $1.3216(9)$ & 0.0369 (19) \\
\hline
\end{tabular}




$\begin{array}{lllll}\text { H24 } & 0.7535 & 0.2998 & 1.3900 & 0.044^{*} \\ \text { C25 } & 0.9411(10) & 0.2544(6) & 1.3561(9) & 0.046(2) \\ \text { H25A } & 0.9761 & 0.2990 & 1.3973 & 0.055^{*} \\ \text { H25B } & 0.9475 & 0.2179 & 1.4302 & 0.055^{*} \\ \text { C26 } & 1.0352(10) & 0.2341(5) & 1.2404(9) & 0.039(2) \\ \text { H26A } & 1.0366 & 0.1821 & 1.2321 & 0.047^{*} \\ \text { H26B } & 1.1303 & 0.2500 & 1.2633 & 0.047^{*} \\ \text { C27 } & 1.0758(8) & 0.2501(4) & 0.9691(8) & 0.0271(16) \\ \text { H27 } & 1.1051 & 0.1996 & 0.9753 & 0.033^{*} \\ \text { C28 } & 1.2069(8) & 0.2936(5) & 0.9501(9) & 0.0333(18) \\ \text { H28A } & 1.1839 & 0.3442 & 0.9612 & 0.040^{*} \\ \text { H28B } & 1.2740 & 0.2809 & 1.0248 & 0.040^{*} \\ \text { C29 } & 1.2738(8) & 0.2823(5) & 0.8075(9) & 0.0369(19) \\ \text { H29A } & 1.3313 & 0.2391 & 0.8104 & 0.044^{*} \\ \text { H29B } & 1.3356 & 0.3226 & 0.7862 & 0.044^{*} \\ \text { C30 } & 1.1624(8) & 0.2751(5) & 0.6870(8) & 0.0304(17) \\ \text { H30 } & 1.1956 & 0.2951 & 0.5956 & 0.036^{*} \\ \text { C31 } & 1.1162(8) & 0.1991(4) & 0.6727(8) & 0.0316(17) \\ \text { C32 } & 0.9801(8) & 0.1890(4) & 0.7541(8) & 0.0285(16) \\ \text { H32A } & 0.9000 & 0.1853 & 0.6893 & 0.034^{*} \\ \text { H32B } & 0.9837 & 0.1465 & 0.8145 & 0.034^{*} \\ \text { C33 } & 0.9726(7) & 0.2559(4) & 0.8422(8) & 0.0245(15) \\ \text { C34 } & 1.0251(8) & 0.3109(4) & 0.7386(8) & 0.0286(16) \\ \text { H34A } & 1.0436 & 0.3566 & 0.7856 & 0.034^{*} \\ \text { H34B } & 0.9592 & 0.3182 & 0.6601 & 0.034^{*} \\ \text { C35 } & 0.8283(7) & 0.2725(4) & 0.9086(7) & 0.0254(15) \\ \text { H35 } & 0.8138 & 0.3247 & 0.9067 & 0.031^{*} \\ \text { C36 } & 0.6119(9) & 0.3093(5) & 1.1440(10) & 0.040(2) \\ \text { H36A } & 0.6011 & 0.3314 & 1.0514 & 0.060^{*} \\ \text { H36B } & 0.5765 & 0.3411 & 1.2171 & 0.060^{*} \\ \text { H36C } & 0.5598 & 0.2649 & 1.1464 & 0.060^{*} \\ \text { C37 } & 1.1780(10) & 0.1493(5) & 0.6011(11) & 0.048(2) \\ \text { H37A } & 1.2610 & 0.1592 & 0.5510 & 0.058^{*} \\ \text { H37B } & 1.1399 & 0.1031 & 0.5992 & 0.058^{*} \\ \text { C38 } & 0.7126(7) & 0.2382(4) & 0.8290(8) & 0.0255(16) \\ \text { H6WA } & -0.060(5) & 0.500(3) & 0.074(7) & 0.060(18)^{*} \\ \text { H12B } & 0.569(6) & 0.213(5) & 0.477(7) & 0.04(3)^{*} \\ \text { H12A } & 0.441(8) & 0.246(7) & 0.485(8) & 0.09(5)^{*} \\ \text { H6WB } & 0.073(7) & 0.501(4) & 0.017(15) & 0.09(5)^{*} \\ & & & & \end{array}$

Atomic displacement parameters $\left(\AA^{2}\right)$

\begin{tabular}{lllllll}
\hline & $U^{11}$ & $U^{22}$ & $U^{33}$ & $U^{12}$ & $U^{13}$ & $U^{23}$ \\
\hline O1 & $0.030(3)$ & $0.030(3)$ & $0.032(3)$ & $0.003(2)$ & $0.002(2)$ & $-0.003(2)$ \\
O2 & $0.042(3)$ & $0.023(3)$ & $0.037(3)$ & $-0.004(3)$ & $0.005(2)$ & $0.000(2)$ \\
O3 & $0.043(3)$ & $0.033(3)$ & $0.044(3)$ & $0.000(3)$ & $-0.005(3)$ & $0.003(3)$ \\
O4 & $0.040(3)$ & $0.030(3)$ & $0.034(3)$ & $0.004(2)$ & $0.007(2)$ & $0.004(2)$ \\
O5 & $0.038(3)$ & $0.030(3)$ & $0.045(3)$ & $0.001(3)$ & $0.008(3)$ & $0.006(3)$
\end{tabular}




\begin{tabular}{|c|c|c|c|c|c|c|}
\hline O6 & $0.027(3)$ & $0.048(4)$ & $0.038(3)$ & $0.000(3)$ & $0.003(3)$ & 0.003 \\
\hline $\mathrm{O} 7$ & $0.031(3)$ & $0.026(3)$ & $0.035(3)$ & $-0.006(2)$ & $0.000(2)$ & $-0.002(2)$ \\
\hline O8 & $0.051(4)$ & $0.024(3)$ & $0.040(3)$ & $0.004(3)$ & $0.003(3)$ & $-0.001(2)$ \\
\hline O9 & $0.043(3)$ & $0.032(3)$ & $0.043(3)$ & $-0.002(3)$ & $0.008(3)$ & $0.002(3)$ \\
\hline $\mathrm{O} 10$ & $0.039(3)$ & 0.040 & $0.041(3)$ & -0.003 & $-0.006(3)$ & $0.001(3)$ \\
\hline $\mathrm{O} 11$ & $0.039(3)$ & $0.028(3)$ & 0.045 & $-0.006(3)$ & $0.000(3)$ & $0.000(3)$ \\
\hline $\mathrm{O} 12$ & $0.028(3)$ & $0.049(4)$ & $0.038(3)$ & $0.001(3)$ & $0.003(3)$ & -0.001 \\
\hline $\mathrm{C} 1$ & $0.038(4)$ & $0.021(4)$ & $0.027(4)$ & $-0.001(3)$ & $0.001(3)$ & $-0.002(3)$ \\
\hline $\mathrm{C} 2$ & $0.028(4)$ & $0.024(4)$ & $0.026(4)$ & $0.004(3)$ & $-0.001(3)$ & $-0.001(3)$ \\
\hline $\mathrm{C} 3$ & $0.029(4)$ & $0.024(4)$ & $0.024(4)$ & $-0.002(3)$ & $-0.002(3)$ & $0.000(3)$ \\
\hline $\mathrm{C} 4$ & $0.033(4)$ & $0.035(5)$ & $0.023(4)$ & $-0.007(3)$ & $0.000(3)$ & $0.003(3)$ \\
\hline $\mathrm{C} 5$ & $0.045(5)$ & $0.037(5)$ & $0.026(4)$ & $0.005(4)$ & $0.001(3)$ & -0.001 \\
\hline C6 & $0.048(5)$ & $0.036(5)$ & $0.032(4)$ & $0.012(4)$ & $0.011(4)$ & $0.012(4)$ \\
\hline $\mathrm{C} 7$ & $0.037(5)$ & $0.042(5)$ & $0.029(4)$ & $0.000(4)$ & $0.004(3)$ & $0.001(4)$ \\
\hline $\mathrm{C} 8$ & $0.030(4)$ & $0.038(4)$ & $0.030(4)$ & $-0.006(3)$ & $-0.004(3)$ & $0.001(3)$ \\
\hline C9 & $0.028(4)$ & $0.050(6)$ & $0.038(5)$ & $0.003(4)$ & $0.001(3)$ & $-0.008(4)$ \\
\hline $\mathrm{C} 10$ & $0.026(4)$ & $0.051(5)$ & $0.055(5)$ & $0.004(4)$ & $-0.017(4)$ & $-0.011(5)$ \\
\hline $\mathrm{C} 11$ & $0.038(4)$ & $0.027(4)$ & $0.034(4)$ & $-0.001(3)$ & $-0.010(3)$ & $0.004(3)$ \\
\hline $\mathrm{C} 12$ & $0.036(4)$ & $0.032(4)$ & $0.029(4)$ & $-0.007(4)$ & $0.000(3)$ & $0.000(3)$ \\
\hline $\mathrm{C} 13$ & $0.038(4)$ & $0.026(4)$ & $0.029(4)$ & $-0.006(3)$ & $-0.003(3)$ & $0.001(3)$ \\
\hline $\mathrm{C} 14$ & $0.026(4)$ & $0.024(4)$ & $0.028(4)$ & $0.002(3)$ & -0.001 & $0.001(3)$ \\
\hline $\mathrm{C} 15$ & $0.030(4)$ & $0.032(4)$ & $0.033(4)$ & $0.003(3)$ & $0.000(3)$ & $0.006(3)$ \\
\hline $\mathrm{C} 16$ & $0.027(4)$ & $0.023(4)$ & $0.023(3)$ & -0.010 & -0.005 & $0.002(3)$ \\
\hline $\mathrm{C} 17$ & $0.031(4)$ & $0.042(5)$ & $0.038(4)$ & $-0.004(4)$ & $-0.004(3)$ & -0.004 \\
\hline C18 & $0.050(6)$ & $0.042(5)$ & $0.068(7)$ & $-0.009(5)$ & $-0.012(5)$ & $-0.011(5)$ \\
\hline C19 & $0.027(4)$ & $0.026(4)$ & $0.028(4)$ & $-0.003(3)$ & -0.001 & $-0.001(3)$ \\
\hline $\mathrm{C} 20$ & $0.030(4)$ & $0.026(4)$ & $0.026(4)$ & $-0.004(3)$ & $-0.003(3)$ & $0.001(3)$ \\
\hline $\mathrm{C} 21$ & $0.031(4)$ & $0.022(4)$ & $0.023(4)$ & $-0.004(3)$ & $-0.002(3)$ & $0.002(3)$ \\
\hline $\mathrm{C} 22$ & $0.030(4)$ & $0.027(4)$ & $0.031(4)$ & $-0.003(3)$ & $0.008(3)$ & $0.002(3)$ \\
\hline $\mathrm{C} 23$ & $0.037(4)$ & $0.028(4)$ & $0.023(4)$ & $0.005(3)$ & $-0.003(3)$ & $-0.004(3)$ \\
\hline $\mathrm{C} 24$ & $0.056(5)$ & $0.026(4)$ & $0.029(4)$ & $0.001(4)$ & $0.003(4)$ & $-0.005(3)$ \\
\hline $\mathrm{C} 25$ & $0.055(6)$ & $0.056(6)$ & $0.027(4)$ & $-0.014(5)$ & $-0.004(4)$ & $0.010(4)$ \\
\hline $\mathrm{C} 26$ & $0.048(5)$ & $0.038(5)$ & $0.032(4)$ & $0.008(4)$ & $-0.004(4)$ & $0.006(4)$ \\
\hline $\mathrm{C} 27$ & $0.031(4)$ & $0.023(4)$ & $0.028(4)$ & $0.005(3)$ & $0.000(3)$ & $0.001(3)$ \\
\hline $\mathrm{C} 28$ & $0.025(4)$ & $0.038(5)$ & $0.037(4)$ & $-0.006(3)$ & $-0.005(3)$ & $0.003(4)$ \\
\hline $\mathrm{C} 29$ & $0.026(4)$ & $0.036(5)$ & $0.049(5)$ & $-0.001(3)$ & $0.008(3)$ & $0.002(4)$ \\
\hline $\mathrm{C} 30$ & $0.028(4)$ & $0.036(4)$ & $0.028(4)$ & $0.003(3)$ & $0.007(3)$ & 0.003 \\
\hline $\mathrm{C} 31$ & $0.037(4)$ & 0.025 & $0.033(4)$ & $0.002(3)$ & $0.004(3)$ & $0.002(3)$ \\
\hline $\mathrm{C} 32$ & $0.027(4)$ & $0.024(4)$ & $0.034(4)$ & -0.003 & $0.002(3)$ & $-0.002(3)$ \\
\hline C33 & $0.020(3)$ & $0.026(4)$ & $0.027(4)$ & $0.005(3)$ & 0.003 & 0.000 \\
\hline C34 & $0.030(4)$ & $0.020(4)$ & $0.035(4)$ & $0.005(3)$ & 0.002 & $0.002(3)$ \\
\hline $\mathrm{C} 35$ & $0.022(3)$ & $0.027(4)$ & $0.027(4)$ & $-0.002(3)$ & 0.005 & 0.003 \\
\hline $\mathrm{C} 36$ & $0.039(5)$ & $0.039(5)$ & $0.042(5)$ & $0.006(4)$ & $0.010(4)$ & $-0.006(4)$ \\
\hline $\mathrm{C} 37$ & $0.041(5)$ & $0.039(5)$ & $0.065(6)$ & $0.007(4)$ & $0.020(5)$ & $-0.009(5)$ \\
\hline C38 & $0.020(3)$ & $0.029(4)$ & $0.028(4)$ & $0.002(3)$ & $0.004(3)$ & -0.001 \\
\hline
\end{tabular}


Geometric parameters $\left(\AA,{ }^{\circ}\right)$

\begin{tabular}{|c|c|c|c|}
\hline $\mathrm{O} 1-\mathrm{C} 4$ & $1.368(9)$ & $\mathrm{C} 14-\mathrm{C} 16$ & $1.558(10)$ \\
\hline $\mathrm{O} 1-\mathrm{C} 1$ & $1.503(9)$ & $\mathrm{C} 15-\mathrm{H} 15 \mathrm{~A}$ & 0.9900 \\
\hline $\mathrm{O} 2-\mathrm{C} 4$ & $1.198(10)$ & $\mathrm{C} 15-\mathrm{H} 15 \mathrm{~B}$ & 0.9900 \\
\hline $\mathrm{O} 3-\mathrm{C} 5$ & $1.434(10)$ & $\mathrm{C} 16-\mathrm{C} 19$ & $1.509(10)$ \\
\hline $\mathrm{O} 3-\mathrm{H} 3$ & 0.8400 & $\mathrm{C} 16-\mathrm{H} 16$ & 1.0000 \\
\hline $\mathrm{O} 4-\mathrm{C} 19$ & $1.309(9)$ & C17-H17A & 0.9800 \\
\hline $\mathrm{O} 4-\mathrm{H} 4$ & 0.8400 & C17-H17B & 0.9800 \\
\hline $\mathrm{O} 5-\mathrm{C} 19$ & $1.224(10)$ & $\mathrm{C} 17-\mathrm{H} 17 \mathrm{C}$ & 0.9800 \\
\hline O6-H6WA & $0.82(2)$ & $\mathrm{C} 18-\mathrm{H} 18 \mathrm{~A}$ & 0.9500 \\
\hline O6-H6WB & $0.82(2)$ & $\mathrm{C} 18-\mathrm{H} 18 \mathrm{~B}$ & 0.9500 \\
\hline $\mathrm{O} 7-\mathrm{C} 23$ & $1.353(9)$ & $\mathrm{C} 20-\mathrm{C} 27$ & $1.511(10)$ \\
\hline $\mathrm{O} 7-\mathrm{C} 20$ & $1.509(9)$ & $\mathrm{C} 20-\mathrm{C} 26$ & $1.523(11)$ \\
\hline $\mathrm{O} 8-\mathrm{C} 23$ & $1.216(9)$ & $\mathrm{C} 20-\mathrm{C} 21$ & $1.526(10)$ \\
\hline $\mathrm{O} 9-\mathrm{C} 24$ & $1.432(10)$ & $\mathrm{C} 21-\mathrm{C} 22$ & $1.521(10)$ \\
\hline O9- $\mathrm{H} 9$ & 0.8400 & $\mathrm{C} 21-\mathrm{C} 35$ & $1.555(10)$ \\
\hline $\mathrm{O} 10-\mathrm{C} 38$ & $1.328(9)$ & $\mathrm{C} 21-\mathrm{H} 21$ & 1.0000 \\
\hline $\mathrm{O} 10-\mathrm{H} 10$ & 0.8400 & $\mathrm{C} 22-\mathrm{C} 36$ & $1.519(11)$ \\
\hline $\mathrm{O} 11-\mathrm{C} 38$ & $1.221(9)$ & $\mathrm{C} 22-\mathrm{C} 23$ & $1.538(11)$ \\
\hline $\mathrm{O} 12-\mathrm{H} 12 \mathrm{~B}$ & $0.83(2)$ & $\mathrm{C} 22-\mathrm{C} 24$ & $1.560(11)$ \\
\hline $\mathrm{O} 12-\mathrm{H} 12 \mathrm{~A}$ & $0.83(2)$ & $\mathrm{C} 24-\mathrm{C} 25$ & $1.497(13)$ \\
\hline $\mathrm{C} 1-\mathrm{C} 7$ & $1.514(11)$ & $\mathrm{C} 24-\mathrm{H} 24$ & 1.0000 \\
\hline $\mathrm{C} 1-\mathrm{C} 8$ & $1.523(11)$ & $\mathrm{C} 25-\mathrm{C} 26$ & $1.476(13)$ \\
\hline $\mathrm{C} 1-\mathrm{C} 2$ & $1.526(11)$ & $\mathrm{C} 25-\mathrm{H} 25 \mathrm{~A}$ & 0.9900 \\
\hline $\mathrm{C} 2-\mathrm{C} 3$ & $1.520(10)$ & $\mathrm{C} 25-\mathrm{H} 25 \mathrm{~B}$ & 0.9900 \\
\hline $\mathrm{C} 2-\mathrm{C} 16$ & $1.527(10)$ & $\mathrm{C} 26-\mathrm{H} 26 \mathrm{~A}$ & 0.9900 \\
\hline $\mathrm{C} 2-\mathrm{H} 2$ & 1.0000 & $\mathrm{C} 26-\mathrm{H} 26 \mathrm{~B}$ & 0.9900 \\
\hline $\mathrm{C} 3-\mathrm{C} 4$ & $1.508(11)$ & $\mathrm{C} 27-\mathrm{C} 28$ & $1.521(11)$ \\
\hline $\mathrm{C} 3-\mathrm{C} 17$ & $1.520(11)$ & $\mathrm{C} 27-\mathrm{C} 33$ & $1.567(10)$ \\
\hline $\mathrm{C} 3-\mathrm{C} 5$ & $1.569(10)$ & $\mathrm{C} 27-\mathrm{H} 27$ & 1.0000 \\
\hline $\mathrm{C} 5-\mathrm{C} 6$ & $1.518(12)$ & $\mathrm{C} 28-\mathrm{C} 29$ & $1.515(11)$ \\
\hline $\mathrm{C} 5-\mathrm{H} 5$ & 1.0000 & $\mathrm{C} 28-\mathrm{H} 28 \mathrm{~A}$ & 0.9900 \\
\hline $\mathrm{C} 6-\mathrm{C} 7$ & $1.473(12)$ & $\mathrm{C} 28-\mathrm{H} 28 \mathrm{~B}$ & 0.9900 \\
\hline C6-H6A & 0.9900 & $\mathrm{C} 29-\mathrm{C} 30$ & $1.576(11)$ \\
\hline C6-H6B & 0.9900 & $\mathrm{C} 29-\mathrm{H} 29 \mathrm{~A}$ & 0.9900 \\
\hline C7-H7A & 0.9900 & $\mathrm{C} 29-\mathrm{H} 29 \mathrm{~B}$ & 0.9900 \\
\hline C7-H7B & 0.9900 & $\mathrm{C} 30-\mathrm{C} 31$ & $1.514(11)$ \\
\hline $\mathrm{C} 8-\mathrm{C} 9$ & $1.521(12)$ & $\mathrm{C} 30-\mathrm{C} 34$ & $1.566(10)$ \\
\hline $\mathrm{C} 8-\mathrm{C} 14$ & $1.576(10)$ & $\mathrm{C} 30-\mathrm{H} 30$ & 1.0000 \\
\hline $\mathrm{C} 8-\mathrm{H} 8$ & 1.0000 & $\mathrm{C} 31-\mathrm{C} 37$ & $1.308(12)$ \\
\hline $\mathrm{C} 9-\mathrm{C} 10$ & $1.555(12)$ & $\mathrm{C} 31-\mathrm{C} 32$ & $1.536(10)$ \\
\hline C9-H9A & 0.9900 & $\mathrm{C} 32-\mathrm{C} 33$ & $1.521(11)$ \\
\hline С9-H9B & 0.9900 & $\mathrm{C} 32-\mathrm{H} 32 \mathrm{~A}$ & 0.9900 \\
\hline $\mathrm{C} 10-\mathrm{C} 11$ & $1.542(13)$ & С $32-\mathrm{H} 32 \mathrm{~B}$ & 0.9900 \\
\hline $\mathrm{C} 10-\mathrm{H} 10 \mathrm{~A}$ & 0.9900 & $\mathrm{C} 33-\mathrm{C} 34$ & $1.520(10)$ \\
\hline $\mathrm{C} 10-\mathrm{H} 10 \mathrm{~B}$ & 0.9900 & $\mathrm{C} 33-\mathrm{C} 35$ & $1.560(9)$ \\
\hline $\mathrm{C} 11-\mathrm{C} 12$ & $1.528(11)$ & C34-H34A & 0.9900 \\
\hline
\end{tabular}




\begin{tabular}{|c|c|c|c|}
\hline $\mathrm{C} 11-\mathrm{C} 15$ & $1.540(11)$ & $\mathrm{C} 34-\mathrm{H} 34 \mathrm{~B}$ & 0.9900 \\
\hline $\mathrm{C} 11-\mathrm{H} 11$ & 1.0000 & $\mathrm{C} 35-\mathrm{C} 38$ & $1.496(10)$ \\
\hline $\mathrm{C} 12-\mathrm{C} 18$ & $1.300(12)$ & $\mathrm{C} 35-\mathrm{H} 35$ & 1.0000 \\
\hline $\mathrm{C} 12-\mathrm{C} 13$ & $1.504(11)$ & $\mathrm{C} 36-\mathrm{H} 36 \mathrm{~A}$ & 0.9800 \\
\hline $\mathrm{C} 13-\mathrm{C} 14$ & $1.530(11)$ & $\mathrm{C} 36-\mathrm{H} 36 \mathrm{~B}$ & 0.9800 \\
\hline C13-H13A & 0.9900 & $\mathrm{C} 36-\mathrm{H} 36 \mathrm{C}$ & 0.9800 \\
\hline C13-H13B & 0.9900 & C37-H37A & 0.9500 \\
\hline $\mathrm{C} 14-\mathrm{C} 15$ & $1.546(10)$ & C37-H37B & 0.9500 \\
\hline $\mathrm{C} 4-\mathrm{O} 1-\mathrm{C} 1$ & $108.5(6)$ & $\mathrm{H} 18 \mathrm{~A}-\mathrm{C} 18-\mathrm{H} 18 \mathrm{~B}$ & 120.0 \\
\hline $\mathrm{C} 5-\mathrm{O} 3-\mathrm{H} 3$ & 109.5 & $\mathrm{O} 5-\mathrm{C} 19-\mathrm{O} 4$ & $123.5(7)$ \\
\hline $\mathrm{C} 19-\mathrm{O} 4-\mathrm{H} 4$ & 109.5 & $\mathrm{O} 5-\mathrm{C} 19-\mathrm{C} 16$ & $124.3(7)$ \\
\hline $\mathrm{H} 6 \mathrm{WA}-\mathrm{O} 6-\mathrm{H} 6 \mathrm{WB}$ & $116(7)$ & $\mathrm{O} 4-\mathrm{C} 19-\mathrm{C} 16$ & $112.2(6)$ \\
\hline $\mathrm{C} 23-\mathrm{O} 7-\mathrm{C} 20$ & $108.2(6)$ & $\mathrm{O} 7-\mathrm{C} 20-\mathrm{C} 27$ & $109.1(6)$ \\
\hline $\mathrm{C} 24-\mathrm{O} 9-\mathrm{H} 9$ & 109.5 & $\mathrm{O} 7-\mathrm{C} 20-\mathrm{C} 26$ & $106.9(6)$ \\
\hline $\mathrm{C} 38-\mathrm{O} 10-\mathrm{H} 10$ & 109.5 & $\mathrm{C} 27-\mathrm{C} 20-\mathrm{C} 26$ & $119.6(7)$ \\
\hline $\mathrm{H} 12 \mathrm{~B}-\mathrm{O} 12-\mathrm{H} 12 \mathrm{~A}$ & $114(8)$ & $\mathrm{O} 7-\mathrm{C} 20-\mathrm{C} 21$ & $101.2(6)$ \\
\hline $\mathrm{O} 1-\mathrm{C} 1-\mathrm{C} 7$ & $108.4(6)$ & $\mathrm{C} 27-\mathrm{C} 20-\mathrm{C} 21$ & $106.9(6)$ \\
\hline $\mathrm{O} 1-\mathrm{C} 1-\mathrm{C} 8$ & $108.8(6)$ & $\mathrm{C} 26-\mathrm{C} 20-\mathrm{C} 21$ & $111.6(6)$ \\
\hline $\mathrm{C} 7-\mathrm{C} 1-\mathrm{C} 8$ & $119.3(7)$ & $\mathrm{C} 22-\mathrm{C} 21-\mathrm{C} 20$ & $101.5(6)$ \\
\hline $\mathrm{O} 1-\mathrm{C} 1-\mathrm{C} 2$ & $100.8(6)$ & $\mathrm{C} 22-\mathrm{C} 21-\mathrm{C} 35$ & $115.2(6)$ \\
\hline $\mathrm{C} 7-\mathrm{C} 1-\mathrm{C} 2$ & $111.2(6)$ & $\mathrm{C} 20-\mathrm{C} 21-\mathrm{C} 35$ & $102.8(6)$ \\
\hline $\mathrm{C} 8-\mathrm{C} 1-\mathrm{C} 2$ & $106.7(6)$ & $\mathrm{C} 22-\mathrm{C} 21-\mathrm{H} 21$ & 112.2 \\
\hline $\mathrm{C} 3-\mathrm{C} 2-\mathrm{C} 1$ & $100.4(6)$ & $\mathrm{C} 20-\mathrm{C} 21-\mathrm{H} 21$ & 112.2 \\
\hline $\mathrm{C} 3-\mathrm{C} 2-\mathrm{C} 16$ & $115.2(6)$ & $\mathrm{C} 35-\mathrm{C} 21-\mathrm{H} 21$ & 112.2 \\
\hline $\mathrm{C} 1-\mathrm{C} 2-\mathrm{C} 16$ & $103.4(6)$ & $\mathrm{C} 36-\mathrm{C} 22-\mathrm{C} 21$ & $117.8(7)$ \\
\hline $\mathrm{C} 3-\mathrm{C} 2-\mathrm{H} 2$ & 112.3 & $\mathrm{C} 36-\mathrm{C} 22-\mathrm{C} 23$ & $112.7(7)$ \\
\hline $\mathrm{C} 1-\mathrm{C} 2-\mathrm{H} 2$ & 112.3 & $\mathrm{C} 21-\mathrm{C} 22-\mathrm{C} 23$ & $99.4(6)$ \\
\hline $\mathrm{C} 16-\mathrm{C} 2-\mathrm{H} 2$ & 112.3 & $\mathrm{C} 36-\mathrm{C} 22-\mathrm{C} 24$ & $112.4(7)$ \\
\hline $\mathrm{C} 4-\mathrm{C} 3-\mathrm{C} 2$ & $101.1(6)$ & $\mathrm{C} 21-\mathrm{C} 22-\mathrm{C} 24$ & $108.6(6)$ \\
\hline $\mathrm{C} 4-\mathrm{C} 3-\mathrm{C} 17$ & $113.1(7)$ & $\mathrm{C} 23-\mathrm{C} 22-\mathrm{C} 24$ & $104.4(6)$ \\
\hline $\mathrm{C} 2-\mathrm{C} 3-\mathrm{C} 17$ & $117.7(6)$ & $\mathrm{O} 8-\mathrm{C} 23-\mathrm{O} 7$ & $120.5(7)$ \\
\hline $\mathrm{C} 4-\mathrm{C} 3-\mathrm{C} 5$ & $105.0(6)$ & $\mathrm{O} 8-\mathrm{C} 23-\mathrm{C} 22$ & $129.2(7)$ \\
\hline $\mathrm{C} 2-\mathrm{C} 3-\mathrm{C} 5$ & $108.7(6)$ & $\mathrm{O} 7-\mathrm{C} 23-\mathrm{C} 22$ & $110.3(6)$ \\
\hline $\mathrm{C} 17-\mathrm{C} 3-\mathrm{C} 5$ & $110.3(6)$ & $\mathrm{O} 9-\mathrm{C} 24-\mathrm{C} 25$ & $113.1(7)$ \\
\hline $\mathrm{O} 2-\mathrm{C} 4-\mathrm{O} 1$ & $120.7(7)$ & $\mathrm{O} 9-\mathrm{C} 24-\mathrm{C} 22$ & $108.1(6)$ \\
\hline $\mathrm{O} 2-\mathrm{C} 4-\mathrm{C} 3$ & $130.1(7)$ & $\mathrm{C} 25-\mathrm{C} 24-\mathrm{C} 22$ & $114.0(7)$ \\
\hline $\mathrm{O} 1-\mathrm{C} 4-\mathrm{C} 3$ & $109.1(7)$ & $\mathrm{O} 9-\mathrm{C} 24-\mathrm{H} 24$ & 107.1 \\
\hline $\mathrm{O} 3-\mathrm{C} 5-\mathrm{C} 6$ & $112.4(7)$ & $\mathrm{C} 25-\mathrm{C} 24-\mathrm{H} 24$ & 107.1 \\
\hline $\mathrm{O} 3-\mathrm{C} 5-\mathrm{C} 3$ & $108.0(6)$ & $\mathrm{C} 22-\mathrm{C} 24-\mathrm{H} 24$ & 107.1 \\
\hline $\mathrm{C} 6-\mathrm{C} 5-\mathrm{C} 3$ & $112.6(7)$ & $\mathrm{C} 26-\mathrm{C} 25-\mathrm{C} 24$ & $117.7(7)$ \\
\hline $\mathrm{O} 3-\mathrm{C} 5-\mathrm{H} 5$ & 107.9 & $\mathrm{C} 26-\mathrm{C} 25-\mathrm{H} 25 \mathrm{~A}$ & 107.9 \\
\hline $\mathrm{C} 6-\mathrm{C} 5-\mathrm{H} 5$ & 107.9 & $\mathrm{C} 24-\mathrm{C} 25-\mathrm{H} 25 \mathrm{~A}$ & 107.9 \\
\hline $\mathrm{C} 3-\mathrm{C} 5-\mathrm{H} 5$ & 107.9 & $\mathrm{C} 26-\mathrm{C} 25-\mathrm{H} 25 \mathrm{~B}$ & 107.9 \\
\hline $\mathrm{C} 7-\mathrm{C} 6-\mathrm{C} 5$ & $115.5(7)$ & $\mathrm{C} 24-\mathrm{C} 25-\mathrm{H} 25 \mathrm{~B}$ & 107.9 \\
\hline $\mathrm{C} 7-\mathrm{C} 6-\mathrm{H} 6 \mathrm{~A}$ & 108.4 & $\mathrm{H} 25 \mathrm{~A}-\mathrm{C} 25-\mathrm{H} 25 \mathrm{~B}$ & 107.2 \\
\hline $\mathrm{C} 5-\mathrm{C} 6-\mathrm{H} 6 \mathrm{~A}$ & 108.4 & $\mathrm{C} 25-\mathrm{C} 26-\mathrm{C} 20$ & $112.3(7)$ \\
\hline $\mathrm{C} 7-\mathrm{C} 6-\mathrm{H} 6 \mathrm{~B}$ & 108.4 & $\mathrm{C} 25-\mathrm{C} 26-\mathrm{H} 26 \mathrm{~A}$ & 109.1 \\
\hline
\end{tabular}


$\mathrm{C} 5-\mathrm{C} 6-\mathrm{H} 6 \mathrm{~B}$

H6A- 6 6- $\mathrm{H} 6 \mathrm{~B}$

$\mathrm{C} 6-\mathrm{C} 7-\mathrm{C} 1$

$\mathrm{C} 6-\mathrm{C} 7-\mathrm{H} 7 \mathrm{~A}$

$\mathrm{C} 1-\mathrm{C} 7-\mathrm{H} 7 \mathrm{~A}$

C6- $\mathrm{C} 7-\mathrm{H} 7 \mathrm{~B}$

$\mathrm{C} 1-\mathrm{C} 7-\mathrm{H} 7 \mathrm{~B}$

$\mathrm{H} 7 \mathrm{~A}-\mathrm{C} 7-\mathrm{H} 7 \mathrm{~B}$

C9- 8 - $-\mathrm{C} 1$

C9- 8 - $-\mathrm{C} 14$

$\mathrm{C} 1-\mathrm{C} 8-\mathrm{C} 14$

C9- 8 - $-\mathrm{H} 8$

$\mathrm{C} 1-\mathrm{C} 8-\mathrm{H} 8$

$\mathrm{C} 14-\mathrm{C} 8-\mathrm{H} 8$

C8-C9-C10

$\mathrm{C} 8-\mathrm{C} 9-\mathrm{H} 9 \mathrm{~A}$

$\mathrm{C} 10-\mathrm{C} 9-\mathrm{H} 9 \mathrm{~A}$

C8-C9-H9B

C10-C9- $\mathrm{H} 9 \mathrm{~B}$

H9A-C9-H9B

$\mathrm{C} 11-\mathrm{C} 10-\mathrm{C} 9$

$\mathrm{C} 11-\mathrm{C} 10-\mathrm{H} 10 \mathrm{~A}$

C9- $\mathrm{C} 10-\mathrm{H} 10 \mathrm{~A}$

C11-C10-H10B

C9- $\mathrm{C} 10-\mathrm{H} 10 \mathrm{~B}$

$\mathrm{H} 10 \mathrm{~A}-\mathrm{C} 10-\mathrm{H} 10 \mathrm{~B}$

$\mathrm{C} 12-\mathrm{C} 11-\mathrm{C} 15$

$\mathrm{C} 12-\mathrm{C} 11-\mathrm{C} 10$

$\mathrm{C} 15-\mathrm{C} 11-\mathrm{C} 10$

$\mathrm{C} 12-\mathrm{C} 11-\mathrm{H} 11$

$\mathrm{C} 15-\mathrm{C} 11-\mathrm{H} 11$

$\mathrm{C} 10-\mathrm{C} 11-\mathrm{H} 11$

$\mathrm{C} 18-\mathrm{C} 12-\mathrm{C} 13$

$\mathrm{C} 18-\mathrm{C} 12-\mathrm{C} 11$

$\mathrm{C} 13-\mathrm{C} 12-\mathrm{C} 11$

$\mathrm{C} 12-\mathrm{C} 13-\mathrm{C} 14$

C12-C13-H13A

C14-C13-H13A

C12-C13-H13B

C14-C13-H13B

H13A-C13-H13B

C13-C14-C15

$\mathrm{C} 13-\mathrm{C} 14-\mathrm{C} 16$

$\mathrm{C} 15-\mathrm{C} 14-\mathrm{C} 16$

$\mathrm{C} 13-\mathrm{C} 14-\mathrm{C} 8$

$\mathrm{C} 15-\mathrm{C} 14-\mathrm{C} 8$

$\mathrm{C} 16-\mathrm{C} 14-\mathrm{C} 8$

$\mathrm{C} 11-\mathrm{C} 15-\mathrm{C} 14$
108.4

107.5

112.4 (7)

109.1

109.1

109.1

109.1

107.9

$114.8(7)$

$114.1(6)$

$104.9(6)$

107.5

107.5

107.5

$111.3(7)$

109.4

109.4

109.4

109.4

108.0

$111.6(7)$

109.3

109.3

109.3

109.3

108.0

101.7 (6)

$112.1(7)$

$110.5(7)$

110.7

110.7

110.7

$126.8(9)$

$125.5(8)$

$107.6(6)$

$104.6(6)$

110.8

110.8

110.8

110.8

108.9

$100.4(6)$

$116.7(6)$

$115.3(6)$

$109.1(6)$

$109.3(6)$

$105.8(6)$

99.9 (6)
$\mathrm{C} 20-\mathrm{C} 26-\mathrm{H} 26 \mathrm{~A}$

$\mathrm{C} 25-\mathrm{C} 26-\mathrm{H} 26 \mathrm{~B}$

$\mathrm{C} 20-\mathrm{C} 26-\mathrm{H} 26 \mathrm{~B}$

$\mathrm{H} 26 \mathrm{~A}-\mathrm{C} 26-\mathrm{H} 26 \mathrm{~B}$

$\mathrm{C} 20-\mathrm{C} 27-\mathrm{C} 28$

$\mathrm{C} 20-\mathrm{C} 27-\mathrm{C} 33$

$\mathrm{C} 28-\mathrm{C} 27-\mathrm{C} 33$

$\mathrm{C} 20-\mathrm{C} 27-\mathrm{H} 27$

$\mathrm{C} 28-\mathrm{C} 27-\mathrm{H} 27$

$\mathrm{C} 33-\mathrm{C} 27-\mathrm{H} 27$

$\mathrm{C} 29-\mathrm{C} 28-\mathrm{C} 27$

$\mathrm{C} 29-\mathrm{C} 28-\mathrm{H} 28 \mathrm{~A}$

$\mathrm{C} 27-\mathrm{C} 28-\mathrm{H} 28 \mathrm{~A}$

$\mathrm{C} 29-\mathrm{C} 28-\mathrm{H} 28 \mathrm{~B}$

C27-C $28-\mathrm{H} 28 \mathrm{~B}$

$\mathrm{H} 28 \mathrm{~A}-\mathrm{C} 28-\mathrm{H} 28 \mathrm{~B}$

C28-C29-C30

$\mathrm{C} 28-\mathrm{C} 29-\mathrm{H} 29 \mathrm{~A}$

C $30-\mathrm{C} 29-\mathrm{H} 29 \mathrm{~A}$

C28-C29- $\mathrm{H} 29 \mathrm{~B}$

C30-C29-H29B

$\mathrm{H} 29 \mathrm{~A}-\mathrm{C} 29-\mathrm{H} 29 \mathrm{~B}$

C31-C30-C34

$\mathrm{C} 31-\mathrm{C} 30-\mathrm{C} 29$

C34-C30-C29

$\mathrm{C} 31-\mathrm{C} 30-\mathrm{H} 30$

$\mathrm{C} 34-\mathrm{C} 30-\mathrm{H} 30$

$\mathrm{C} 29-\mathrm{C} 30-\mathrm{H} 30$

$\mathrm{C} 37-\mathrm{C} 31-\mathrm{C} 30$

$\mathrm{C} 37-\mathrm{C} 31-\mathrm{C} 32$

$\mathrm{C} 30-\mathrm{C} 31-\mathrm{C} 32$

$\mathrm{C} 33-\mathrm{C} 32-\mathrm{C} 31$

C $33-\mathrm{C} 32-\mathrm{H} 32 \mathrm{~A}$

$\mathrm{C} 31-\mathrm{C} 32-\mathrm{H} 32 \mathrm{~A}$

C $33-\mathrm{C} 32-\mathrm{H} 32 \mathrm{~B}$

$\mathrm{C} 31-\mathrm{C} 32-\mathrm{H} 32 \mathrm{~B}$

$\mathrm{H} 32 \mathrm{~A}-\mathrm{C} 32-\mathrm{H} 32 \mathrm{~B}$

C34-C33-C32

C34-C33-C35

$\mathrm{C} 32-\mathrm{C} 33-\mathrm{C} 35$

$\mathrm{C} 34-\mathrm{C} 33-\mathrm{C} 27$

$\mathrm{C} 32-\mathrm{C} 33-\mathrm{C} 27$

$\mathrm{C} 35-\mathrm{C} 33-\mathrm{C} 27$

$\mathrm{C} 33-\mathrm{C} 34-\mathrm{C} 30$

C33-C $34-\mathrm{H} 34 \mathrm{~A}$

$\mathrm{C} 30-\mathrm{C} 34-\mathrm{H} 34 \mathrm{~A}$

C33-C $34-\mathrm{H} 34 \mathrm{~B}$

$\mathrm{C} 30-\mathrm{C} 34-\mathrm{H} 34 \mathrm{~B}$
109.1

109.1

109.1

107.9

$115.8(6)$

$106.0(6)$

$113.5(6)$

107.0

107.0

107.0

$112.5(7)$

109.1

109.1

109.1

109.1

107.8

111.7 (6)

109.3

109.3

109.3

109.3

107.9

101.1 (6)

$110.4(7)$

108.2 (6)

112.2

112.2

112.2

$126.9(8)$

$124.1(8)$

108.9 (6)

102.3 (6)

111.3

111.3

111.3

111.3

109.2

$101.6(6)$

$114.9(6)$

$115.6(6)$

109.5 (6)

109.4 (6)

105.7 (6)

100.7 (6)

111.6

111.6

111.6

111.6 


\begin{tabular}{|c|c|c|c|}
\hline $\mathrm{C} 11-\mathrm{C} 15-\mathrm{H} 15 \mathrm{~A}$ & 111.8 & $\mathrm{H} 34 \mathrm{~A}-\mathrm{C} 34-\mathrm{H} 34 \mathrm{~B}$ & 109.4 \\
\hline $\mathrm{C} 14-\mathrm{C} 15-\mathrm{H} 15 \mathrm{~A}$ & 111.8 & $\mathrm{C} 38-\mathrm{C} 35-\mathrm{C} 21$ & $114.5(6)$ \\
\hline $\mathrm{C} 11-\mathrm{C} 15-\mathrm{H} 15 \mathrm{~B}$ & 111.8 & $\mathrm{C} 38-\mathrm{C} 35-\mathrm{C} 33$ & $112.0(6)$ \\
\hline $\mathrm{C} 14-\mathrm{C} 15-\mathrm{H} 15 \mathrm{~B}$ & 111.8 & $\mathrm{C} 21-\mathrm{C} 35-\mathrm{C} 33$ & $104.8(6)$ \\
\hline $\mathrm{H} 15 \mathrm{~A}-\mathrm{C} 15-\mathrm{H} 15 \mathrm{~B}$ & 109.5 & $\mathrm{C} 38-\mathrm{C} 35-\mathrm{H} 35$ & 108.4 \\
\hline $\mathrm{C} 19-\mathrm{C} 16-\mathrm{C} 2$ & $116.4(6)$ & $\mathrm{C} 21-\mathrm{C} 35-\mathrm{H} 35$ & 108.4 \\
\hline $\mathrm{C} 19-\mathrm{C} 16-\mathrm{C} 14$ & $112.4(6)$ & $\mathrm{C} 33-\mathrm{C} 35-\mathrm{H} 35$ & 108.4 \\
\hline $\mathrm{C} 2-\mathrm{C} 16-\mathrm{C} 14$ & $105.8(6)$ & $\mathrm{C} 22-\mathrm{C} 36-\mathrm{H} 36 \mathrm{~A}$ & 109.5 \\
\hline $\mathrm{C} 19-\mathrm{C} 16-\mathrm{H} 16$ & 107.3 & $\mathrm{C} 22-\mathrm{C} 36-\mathrm{H} 36 \mathrm{~B}$ & 109.5 \\
\hline $\mathrm{C} 2-\mathrm{C} 16-\mathrm{H} 16$ & 107.3 & $\mathrm{H} 36 \mathrm{~A}-\mathrm{C} 36-\mathrm{H} 36 \mathrm{~B}$ & 109.5 \\
\hline $\mathrm{C} 14-\mathrm{C} 16-\mathrm{H} 16$ & 107.3 & $\mathrm{C} 22-\mathrm{C} 36-\mathrm{H} 36 \mathrm{C}$ & 109.5 \\
\hline $\mathrm{C} 3-\mathrm{C} 17-\mathrm{H} 17 \mathrm{~A}$ & 109.5 & $\mathrm{H} 36 \mathrm{~A}-\mathrm{C} 36-\mathrm{H} 36 \mathrm{C}$ & 109.5 \\
\hline C3-C17-H17B & 109.5 & $\mathrm{H} 36 \mathrm{~B}-\mathrm{C} 36-\mathrm{H} 36 \mathrm{C}$ & 109.5 \\
\hline $\mathrm{H} 17 \mathrm{~A}-\mathrm{C} 17-\mathrm{H} 17 \mathrm{~B}$ & 109.5 & $\mathrm{C} 31-\mathrm{C} 37-\mathrm{H} 37 \mathrm{~A}$ & 120.0 \\
\hline $\mathrm{C} 3-\mathrm{C} 17-\mathrm{H} 17 \mathrm{C}$ & 109.5 & C $31-\mathrm{C} 37-\mathrm{H} 37 \mathrm{~B}$ & 120.0 \\
\hline $\mathrm{H} 17 \mathrm{~A}-\mathrm{C} 17-\mathrm{H} 17 \mathrm{C}$ & 109.5 & $\mathrm{H} 37 \mathrm{~A}-\mathrm{C} 37-\mathrm{H} 37 \mathrm{~B}$ & 120.0 \\
\hline $\mathrm{H} 17 \mathrm{~B}-\mathrm{C} 17-\mathrm{H} 17 \mathrm{C}$ & 109.5 & $\mathrm{O} 11-\mathrm{C} 38-\mathrm{O} 10$ & $123.4(7)$ \\
\hline $\mathrm{C} 12-\mathrm{C} 18-\mathrm{H} 18 \mathrm{~A}$ & 120.0 & $\mathrm{O} 11-\mathrm{C} 38-\mathrm{C} 35$ & $124.7(7)$ \\
\hline $\mathrm{C} 12-\mathrm{C} 18-\mathrm{H} 18 \mathrm{~B}$ & 120.0 & $\mathrm{O} 10-\mathrm{C} 38-\mathrm{C} 35$ & $111.9(6)$ \\
\hline $\mathrm{C} 4-\mathrm{O} 1-\mathrm{C} 1-\mathrm{C} 7$ & $-87.7(7)$ & $\mathrm{C} 23-\mathrm{O} 7-\mathrm{C} 20-\mathrm{C} 27$ & $139.9(6)$ \\
\hline $\mathrm{C} 4-\mathrm{O} 1-\mathrm{C} 1-\mathrm{C} 8$ & $141.1(6)$ & $\mathrm{C} 23-\mathrm{O} 7-\mathrm{C} 20-\mathrm{C} 26$ & $-89.5(7)$ \\
\hline $\mathrm{C} 4-\mathrm{O} 1-\mathrm{C} 1-\mathrm{C} 2$ & $29.1(7)$ & $\mathrm{C} 23-\mathrm{O} 7-\mathrm{C} 20-\mathrm{C} 21$ & $27.4(7)$ \\
\hline $\mathrm{O} 1-\mathrm{C} 1-\mathrm{C} 2-\mathrm{C} 3$ & $-42.7(7)$ & $\mathrm{O} 7-\mathrm{C} 20-\mathrm{C} 21-\mathrm{C} 22$ & $-42.2(6)$ \\
\hline $\mathrm{C} 7-\mathrm{C} 1-\mathrm{C} 2-\mathrm{C} 3$ & $72.0(7)$ & $\mathrm{C} 27-\mathrm{C} 20-\mathrm{C} 21-\mathrm{C} 22$ & $-156.4(6)$ \\
\hline $\mathrm{C} 8-\mathrm{C} 1-\mathrm{C} 2-\mathrm{C} 3$ & $-156.3(6)$ & $\mathrm{C} 26-\mathrm{C} 20-\mathrm{C} 21-\mathrm{C} 22$ & $71.2(8)$ \\
\hline $\mathrm{O} 1-\mathrm{C} 1-\mathrm{C} 2-\mathrm{C} 16$ & $76.5(6)$ & $\mathrm{O} 7-\mathrm{C} 20-\mathrm{C} 21-\mathrm{C} 35$ & $77.2(6)$ \\
\hline $\mathrm{C} 7-\mathrm{C} 1-\mathrm{C} 2-\mathrm{C} 16$ & $-168.8(6)$ & $\mathrm{C} 27-\mathrm{C} 20-\mathrm{C} 21-\mathrm{C} 35$ & $-36.9(7)$ \\
\hline $\mathrm{C} 8-\mathrm{C} 1-\mathrm{C} 2-\mathrm{C} 16$ & $-37.1(7)$ & $\mathrm{C} 26-\mathrm{C} 20-\mathrm{C} 21-\mathrm{C} 35$ & $-169.4(6)$ \\
\hline $\mathrm{C} 1-\mathrm{C} 2-\mathrm{C} 3-\mathrm{C} 4$ & $40.9(7)$ & $\mathrm{C} 20-\mathrm{C} 21-\mathrm{C} 22-\mathrm{C} 36$ & $162.4(7)$ \\
\hline $\mathrm{C} 16-\mathrm{C} 2-\mathrm{C} 3-\mathrm{C} 4$ & $-69.3(7)$ & $\mathrm{C} 35-\mathrm{C} 21-\mathrm{C} 22-\mathrm{C} 36$ & $52.2(9)$ \\
\hline $\mathrm{C} 1-\mathrm{C} 2-\mathrm{C} 3-\mathrm{C} 17$ & $164.6(7)$ & $\mathrm{C} 20-\mathrm{C} 21-\mathrm{C} 22-\mathrm{C} 23$ & $40.4(7)$ \\
\hline $\mathrm{C} 16-\mathrm{C} 2-\mathrm{C} 3-\mathrm{C} 17$ & $54.3(9)$ & $\mathrm{C} 35-\mathrm{C} 21-\mathrm{C} 22-\mathrm{C} 23$ & $-69.8(7)$ \\
\hline $\mathrm{C} 1-\mathrm{C} 2-\mathrm{C} 3-\mathrm{C} 5$ & $-69.2(7)$ & $\mathrm{C} 20-\mathrm{C} 21-\mathrm{C} 22-\mathrm{C} 24$ & $-68.4(7)$ \\
\hline $\mathrm{C} 16-\mathrm{C} 2-\mathrm{C} 3-\mathrm{C} 5$ & $-179.4(6)$ & $\mathrm{C} 35-\mathrm{C} 21-\mathrm{C} 22-\mathrm{C} 24$ & $-178.6(6)$ \\
\hline $\mathrm{C} 1-\mathrm{O} 1-\mathrm{C} 4-\mathrm{O} 2$ & $179.9(7)$ & $\mathrm{C} 20-\mathrm{O} 7-\mathrm{C} 23-\mathrm{O} 8$ & $179.3(7)$ \\
\hline $\mathrm{C} 1-\mathrm{O} 1-\mathrm{C} 4-\mathrm{C} 3$ & $-3.0(8)$ & $\mathrm{C} 20-\mathrm{O} 7-\mathrm{C} 23-\mathrm{C} 22$ & $-1.4(8)$ \\
\hline $\mathrm{C} 2-\mathrm{C} 3-\mathrm{C} 4-\mathrm{O} 2$ & $152.2(8)$ & $\mathrm{C} 36-\mathrm{C} 22-\mathrm{C} 23-\mathrm{O} 8$ & $28.5(11)$ \\
\hline $\mathrm{C} 17-\mathrm{C} 3-\mathrm{C} 4-\mathrm{O} 2$ & $25.5(11)$ & $\mathrm{C} 21-\mathrm{C} 22-\mathrm{C} 23-\mathrm{O} 8$ & $154.1(8)$ \\
\hline $\mathrm{C} 5-\mathrm{C} 3-\mathrm{C} 4-\mathrm{O} 2$ & $-94.7(10)$ & $\mathrm{C} 24-\mathrm{C} 22-\mathrm{C} 23-\mathrm{O} 8$ & $-93.8(9)$ \\
\hline $\mathrm{C} 2-\mathrm{C} 3-\mathrm{C} 4-\mathrm{O} 1$ & $-24.5(7)$ & $\mathrm{C} 36-\mathrm{C} 22-\mathrm{C} 23-\mathrm{O} 7$ & $-150.7(6)$ \\
\hline $\mathrm{C} 17-\mathrm{C} 3-\mathrm{C} 4-\mathrm{O} 1$ & $-151.3(6)$ & $\mathrm{C} 21-\mathrm{C} 22-\mathrm{C} 23-\mathrm{O} 7$ & $-25.1(7)$ \\
\hline $\mathrm{C} 5-\mathrm{C} 3-\mathrm{C} 4-\mathrm{O} 1$ & $88.5(7)$ & $\mathrm{C} 24-\mathrm{C} 22-\mathrm{C} 23-\mathrm{O} 7$ & $87.0(7)$ \\
\hline $\mathrm{C} 4-\mathrm{C} 3-\mathrm{C} 5-\mathrm{O} 3$ & $-176.7(6)$ & $\mathrm{C} 36-\mathrm{C} 22-\mathrm{C} 24-\mathrm{O} 9$ & $57.8(9)$ \\
\hline $\mathrm{C} 2-\mathrm{C} 3-\mathrm{C} 5-\mathrm{O} 3$ & $-69.2(8)$ & $\mathrm{C} 21-\mathrm{C} 22-\mathrm{C} 24-\mathrm{O} 9$ & $-74.4(8)$ \\
\hline $\mathrm{C} 17-\mathrm{C} 3-\mathrm{C} 5-\mathrm{O} 3$ & $61.3(8)$ & $\mathrm{C} 23-\mathrm{C} 22-\mathrm{C} 24-\mathrm{O} 9$ & $-179.7(6)$ \\
\hline $\mathrm{C} 4-\mathrm{C} 3-\mathrm{C} 5-\mathrm{C} 6$ & $-52.0(9)$ & $\mathrm{C} 36-\mathrm{C} 22-\mathrm{C} 24-\mathrm{C} 25$ & $-175.5(8)$ \\
\hline $\mathrm{C} 2-\mathrm{C} 3-\mathrm{C} 5-\mathrm{C} 6$ & $55.5(9)$ & $\mathrm{C} 21-\mathrm{C} 22-\mathrm{C} 24-\mathrm{C} 25$ & $52.3(9)$ \\
\hline
\end{tabular}




\begin{tabular}{|c|c|}
\hline $\mathrm{C} 17-\mathrm{C} 3-\mathrm{C} 5-\mathrm{C} 6$ & $-174.1(7)$ \\
\hline $\mathrm{O} 3-\mathrm{C} 5-\mathrm{C} 6-\mathrm{C} 7$ & $84.0(9)$ \\
\hline $\mathrm{C} 3-\mathrm{C} 5-\mathrm{C} 6-\mathrm{C} 7$ & $-38.2(10)$ \\
\hline $\mathrm{C} 5-\mathrm{C} 6-\mathrm{C} 7-\mathrm{C} 1$ & $38.8(10)$ \\
\hline $\mathrm{O} 1-\mathrm{C} 1-\mathrm{C} 7-\mathrm{C} 6$ & $52.0(9)$ \\
\hline $\mathrm{C} 8-\mathrm{C} 1-\mathrm{C} 7-\mathrm{C} 6$ & $177.3(7)$ \\
\hline $\mathrm{C} 2-\mathrm{C} 1-\mathrm{C} 7-\mathrm{C} 6$ & $-57.9(9)$ \\
\hline $\mathrm{O} 1-\mathrm{C} 1-\mathrm{C} 8-\mathrm{C} 9$ & $43.9(9)$ \\
\hline $\mathrm{C} 7-\mathrm{C} 1-\mathrm{C} 8-\mathrm{C} 9$ & $-81.1(10)$ \\
\hline $\mathrm{C} 2-\mathrm{C} 1-\mathrm{C} 8-\mathrm{C} 9$ & $151.9(7)$ \\
\hline $\mathrm{O} 1-\mathrm{C} 1-\mathrm{C} 8-\mathrm{C} 14$ & $-82.2(7)$ \\
\hline $\mathrm{C} 7-\mathrm{C} 1-\mathrm{C} 8-\mathrm{C} 14$ & $152.7(7)$ \\
\hline $\mathrm{C} 2-\mathrm{C} 1-\mathrm{C} 8-\mathrm{C} 14$ & $25.7(8)$ \\
\hline $\mathrm{C} 1-\mathrm{C} 8-\mathrm{C} 9-\mathrm{C} 10$ & $-168.4(7)$ \\
\hline $\mathrm{C} 14-\mathrm{C} 8-\mathrm{C} 9-\mathrm{C} 10$ & $-47.2(10)$ \\
\hline $\mathrm{C} 8-\mathrm{C} 9-\mathrm{C} 10-\mathrm{C} 11$ & $38.4(11)$ \\
\hline $\mathrm{C} 9-\mathrm{C} 10-\mathrm{C} 11-\mathrm{C} 12$ & $-89.9(9)$ \\
\hline $\mathrm{C} 9-\mathrm{C} 10-\mathrm{C} 11-\mathrm{C} 15$ & $22.8(10)$ \\
\hline $\mathrm{C} 15-\mathrm{C} 11-\mathrm{C} 12-\mathrm{C} 18$ & $155.2(9)$ \\
\hline $\mathrm{C} 10-\mathrm{C} 11-\mathrm{C} 12-\mathrm{C} 18$ & $-86.7(11)$ \\
\hline $\mathrm{C} 15-\mathrm{C} 11-\mathrm{C} 12-\mathrm{C} 13$ & $-22.0(8)$ \\
\hline $\mathrm{C} 10-\mathrm{C} 11-\mathrm{C} 12-\mathrm{C} 13$ & $96.1(8)$ \\
\hline $\mathrm{C} 18-\mathrm{C} 12-\mathrm{C} 13-\mathrm{C} 14$ & $173.7(9)$ \\
\hline $\mathrm{C} 11-\mathrm{C} 12-\mathrm{C} 13-\mathrm{C} 14$ & $-9.2(8)$ \\
\hline $\mathrm{C} 12-\mathrm{C} 13-\mathrm{C} 14-\mathrm{C} 15$ & $36.5(7)$ \\
\hline $\mathrm{C} 12-\mathrm{C} 13-\mathrm{C} 14-\mathrm{C} 16$ & $161.9(6)$ \\
\hline $\mathrm{C} 12-\mathrm{C} 13-\mathrm{C} 14-\mathrm{C} 8$ & $-78.3(7)$ \\
\hline $\mathrm{C} 9-\mathrm{C} 8-\mathrm{C} 14-\mathrm{C} 13$ & $102.4(8)$ \\
\hline $\mathrm{C} 1-\mathrm{C} 8-\mathrm{C} 14-\mathrm{C} 13$ & $-131.0(7)$ \\
\hline $\mathrm{C} 9-\mathrm{C} 8-\mathrm{C} 14-\mathrm{C} 15$ & $-6.5(9)$ \\
\hline $\mathrm{C} 1-\mathrm{C} 8-\mathrm{C} 14-\mathrm{C} 15$ & 120.0 \\
\hline $\mathrm{C} 9-\mathrm{C} 8-\mathrm{C} 14-\mathrm{C} 16$ & $-131.2(7)$ \\
\hline $\mathrm{C} 1-\mathrm{C} 8-\mathrm{C} 14-\mathrm{C} 16$ & $-4.7(8)$ \\
\hline $\mathrm{C} 12-\mathrm{C} 11-\mathrm{C} 15-\mathrm{C} 14$ & $44.0(7)$ \\
\hline $\mathrm{C} 10-\mathrm{C} 11-\mathrm{C} 15-\mathrm{C} 14$ & $-75.2(8)$ \\
\hline $\mathrm{C} 13-\mathrm{C} 14-\mathrm{C} 15-\mathrm{C} 11$ & $-49.9(7)$ \\
\hline $\mathrm{C} 16-\mathrm{C} 14-\mathrm{C} 15-\mathrm{C} 11$ & $-176.2(6)$ \\
\hline $\mathrm{C} 8-\mathrm{C} 14-\mathrm{C} 15-\mathrm{C} 11$ & $64.8(7)$ \\
\hline $\mathrm{C} 3-\mathrm{C} 2-\mathrm{C} 16-\mathrm{C} 19$ & $-92.6(8)$ \\
\hline $\mathrm{C} 1-\mathrm{C} 2-\mathrm{C} 16-\mathrm{C} 19$ & $158.9(6)$ \\
\hline $\mathrm{C} 3-\mathrm{C} 2-\mathrm{C} 16-\mathrm{C} 14$ & $141.8(6)$ \\
\hline $\mathrm{C} 1-\mathrm{C} 2-\mathrm{C} 16-\mathrm{C} 14$ & $33.4(7)$ \\
\hline $\mathrm{C} 13-\mathrm{C} 14-\mathrm{C} 16-\mathrm{C} 19$ & $-24.3(9)$ \\
\hline $\mathrm{C} 15-\mathrm{C} 14-\mathrm{C} 16-\mathrm{C} 19$ & $93.2(8)$ \\
\hline $\mathrm{C} 8-\mathrm{C} 14-\mathrm{C} 16-\mathrm{C} 19$ & $-145.9(6)$ \\
\hline $\mathrm{C} 13-\mathrm{C} 14-\mathrm{C} 16-\mathrm{C} 2$ & $103.7(7)$ \\
\hline $\mathrm{C} 15-\mathrm{C} 14-\mathrm{C} 16-\mathrm{C} 2$ & $-138.8(7)$ \\
\hline $\mathrm{C} 8-\mathrm{C} 14-\mathrm{C} 16-\mathrm{C} 2$ & $-17.8(8)$ \\
\hline
\end{tabular}

\begin{tabular}{|c|c|}
\hline $\mathrm{C} 23-\mathrm{C} 22-\mathrm{C} 24-\mathrm{C} 25$ & $-53.0(9)$ \\
\hline $\mathrm{O} 9-\mathrm{C} 24-\mathrm{C} 25-\mathrm{C} 26$ & $90.8(10)$ \\
\hline $\mathrm{C} 22-\mathrm{C} 24-\mathrm{C} 25-\mathrm{C} 26$ & $-33.3(12)$ \\
\hline $\mathrm{C} 24-\mathrm{C} 25-\mathrm{C} 26-\mathrm{C} 20$ & $33.1(12)$ \\
\hline $\mathrm{O} 7-\mathrm{C} 20-\mathrm{C} 26-\mathrm{C} 25$ & $56.0(9)$ \\
\hline $\mathrm{C} 27-\mathrm{C} 20-\mathrm{C} 26-\mathrm{C} 25$ & $-179.5(7)$ \\
\hline $\mathrm{C} 21-\mathrm{C} 20-\mathrm{C} 26-\mathrm{C} 25$ & $-53.8(10)$ \\
\hline $\mathrm{O} 7-\mathrm{C} 20-\mathrm{C} 27-\mathrm{C} 28$ & $42.7(8)$ \\
\hline $\mathrm{C} 26-\mathrm{C} 20-\mathrm{C} 27-\mathrm{C} 28$ & $-80.8(9)$ \\
\hline $\mathrm{C} 21-\mathrm{C} 20-\mathrm{C} 27-\mathrm{C} 28$ & $151.3(6)$ \\
\hline $\mathrm{O} 7-\mathrm{C} 20-\mathrm{C} 27-\mathrm{C} 33$ & $-84.2(7)$ \\
\hline $\mathrm{C} 26-\mathrm{C} 20-\mathrm{C} 27-\mathrm{C} 33$ & $152.3(7)$ \\
\hline $\mathrm{C} 21-\mathrm{C} 20-\mathrm{C} 27-\mathrm{C} 33$ & $24.5(8)$ \\
\hline $\mathrm{C} 20-\mathrm{C} 27-\mathrm{C} 28-\mathrm{C} 29$ & $-171.7(7)$ \\
\hline $\mathrm{C} 33-\mathrm{C} 27-\mathrm{C} 28-\mathrm{C} 29$ & $-48.7(9)$ \\
\hline $\mathrm{C} 27-\mathrm{C} 28-\mathrm{C} 29-\mathrm{C} 30$ & $38.6(10)$ \\
\hline $\mathrm{C} 28-\mathrm{C} 29-\mathrm{C} 30-\mathrm{C} 31$ & $-87.4(8)$ \\
\hline $\mathrm{C} 28-\mathrm{C} 29-\mathrm{C} 30-\mathrm{C} 34$ & $22.4(9)$ \\
\hline $\mathrm{C} 34-\mathrm{C} 30-\mathrm{C} 31-\mathrm{C} 37$ & $161.8(9)$ \\
\hline $\mathrm{C} 29-\mathrm{C} 30-\mathrm{C} 31-\mathrm{C} 37$ & $-83.8(11)$ \\
\hline $\mathrm{C} 34-\mathrm{C} 30-\mathrm{C} 31-\mathrm{C} 32$ & $-16.8(8)$ \\
\hline $\mathrm{C} 29-\mathrm{C} 30-\mathrm{C} 31-\mathrm{C} 32$ & $97.5(7)$ \\
\hline $\mathrm{C} 37-\mathrm{C} 31-\mathrm{C} 32-\mathrm{C} 33$ & $167.8(9)$ \\
\hline $\mathrm{C} 30-\mathrm{C} 31-\mathrm{C} 32-\mathrm{C} 33$ & $-13.5(8)$ \\
\hline $\mathrm{C} 31-\mathrm{C} 32-\mathrm{C} 33-\mathrm{C} 34$ & $39.4(7)$ \\
\hline $\mathrm{C} 31-\mathrm{C} 32-\mathrm{C} 33-\mathrm{C} 35$ & $164.5(6)$ \\
\hline $\mathrm{C} 31-\mathrm{C} 32-\mathrm{C} 33-\mathrm{C} 27$ & $-76.3(7)$ \\
\hline $\mathrm{C} 20-\mathrm{C} 27-\mathrm{C} 33-\mathrm{C} 34$ & $122.1(6)$ \\
\hline $\mathrm{C} 28-\mathrm{C} 27-\mathrm{C} 33-\mathrm{C} 34$ & $-6.1(8)$ \\
\hline $\mathrm{C} 20-\mathrm{C} 27-\mathrm{C} 33-\mathrm{C} 32$ & $-127.3(6)$ \\
\hline $\mathrm{C} 28-\mathrm{C} 27-\mathrm{C} 33-\mathrm{C} 32$ & $104.4(7)$ \\
\hline $\mathrm{C} 20-\mathrm{C} 27-\mathrm{C} 33-\mathrm{C} 35$ & $-2.2(8)$ \\
\hline $\mathrm{C} 28-\mathrm{C} 27-\mathrm{C} 33-\mathrm{C} 35$ & $-130.4(7)$ \\
\hline $\mathrm{C} 32-\mathrm{C} 33-\mathrm{C} 34-\mathrm{C} 30$ & $-50.6(7)$ \\
\hline $\mathrm{C} 35-\mathrm{C} 33-\mathrm{C} 34-\mathrm{C} 30$ & $-176.1(6)$ \\
\hline $\mathrm{C} 27-\mathrm{C} 33-\mathrm{C} 34-\mathrm{C} 30$ & $65.1(7)$ \\
\hline $\mathrm{C} 31-\mathrm{C} 30-\mathrm{C} 34-\mathrm{C} 33$ & $40.9(7)$ \\
\hline $\mathrm{C} 29-\mathrm{C} 30-\mathrm{C} 34-\mathrm{C} 33$ & $-75.1(7)$ \\
\hline $\mathrm{C} 22-\mathrm{C} 21-\mathrm{C} 35-\mathrm{C} 38$ & $-92.9(8)$ \\
\hline $\mathrm{C} 20-\mathrm{C} 21-\mathrm{C} 35-\mathrm{C} 38$ & $157.7(6)$ \\
\hline $\mathrm{C} 22-\mathrm{C} 21-\mathrm{C} 35-\mathrm{C} 33$ & $144.0(6)$ \\
\hline $\mathrm{C} 20-\mathrm{C} 21-\mathrm{C} 35-\mathrm{C} 33$ & $34.6(7)$ \\
\hline $\mathrm{C} 34-\mathrm{C} 33-\mathrm{C} 35-\mathrm{C} 38$ & $94.3(8)$ \\
\hline $\mathrm{C} 32-\mathrm{C} 33-\mathrm{C} 35-\mathrm{C} 38$ & $-23.7(9)$ \\
\hline $\mathrm{C} 27-\mathrm{C} 33-\mathrm{C} 35-\mathrm{C} 38$ & $-144.9(6)$ \\
\hline $\mathrm{C} 34-\mathrm{C} 33-\mathrm{C} 35-\mathrm{C} 21$ & $-141.0(7)$ \\
\hline $\mathrm{C} 32-\mathrm{C} 33-\mathrm{C} 35-\mathrm{C} 21$ & $101.1(7)$ \\
\hline $\mathrm{C} 27-\mathrm{C} 33-\mathrm{C} 35-\mathrm{C} 21$ & $-20.2(8)$ \\
\hline
\end{tabular}




$\begin{array}{llll}\mathrm{C} 2-\mathrm{C} 16-\mathrm{C} 19-\mathrm{O} 5 & -29.1(10) & \mathrm{C} 21-\mathrm{C} 35-\mathrm{C} 38-\mathrm{O} 11 & -27.7(10) \\ \mathrm{C} 14-\mathrm{C} 16-\mathrm{C} 19-\mathrm{O} 5 & 93.1(9) & \mathrm{C} 33-\mathrm{C} 35-\mathrm{C} 38-\mathrm{O} 11 & 91.4(9) \\ \mathrm{C} 2-\mathrm{C} 16-\mathrm{C} 19-\mathrm{O} 4 & 152.4(6) & \mathrm{C} 21-\mathrm{C} 35-\mathrm{C} 38-\mathrm{O} 10 & 153.4(6) \\ \mathrm{C} 14-\mathrm{C} 16-\mathrm{C} 19-\mathrm{O} 4 & -85.5(8) & \mathrm{C} 33-\mathrm{C} 35-\mathrm{C} 38-\mathrm{O} 10 & -87.4(8)\end{array}$

Hydrogen-bond geometry $\left(A,{ }^{\circ}\right)$

\begin{tabular}{lllll}
\hline$D-\mathrm{H} \cdots A$ & $D-\mathrm{H}$ & $\mathrm{H} \cdots A$ & $D \cdots A$ & $D-\mathrm{H} \cdots A$ \\
\hline $\mathrm{O} 3-\mathrm{H} 3 \cdots \mathrm{O} 11^{\mathrm{i}}$ & 0.84 & 2.01 & $2.825(8)$ & $163(4)$ \\
$\mathrm{O} 4-\mathrm{H} 4 \cdots \mathrm{O}^{\mathrm{ii}}$ & 0.84 & 1.83 & $2.634(8)$ & $158(6)$ \\
$\mathrm{O} 6-\mathrm{H} 6 W A \cdots{ }^{\mathrm{iii}}$ & $0.82(5)$ & $2.15(6)$ & $2.939(9)$ & $161(6)$ \\
$\mathrm{O} 6-\mathrm{H} 6 W B \cdots \mathrm{O} 4^{\text {iv }}$ & $0.82(5)$ & $1.93(13)$ & $2.634(9)$ & $143(6)$ \\
$\mathrm{O} 10-\mathrm{H} 10 \cdots \mathrm{O} 12$ & $0.83(7)$ & $1.81(6)$ & $2.625(8)$ & $163(4)$ \\
$\mathrm{O} 12-\mathrm{H} 12 A \cdots \mathrm{O} 2$ & $0.82(9)$ & $2.16(11)$ & $2.925(10)$ & $154(10)$ \\
$\mathrm{O} 12-\mathrm{H} 12 B \cdots \mathrm{O} 9^{\text {iv }}$ & $0.82(9)$ & $1.91(6)$ & $2.727(10)$ & $167(2)$ \\
$\mathrm{C} 11-\mathrm{H} 11 \cdots \mathrm{O} 8$ & 1.00 & 2.54 & $3.531(10)$ & $173(7)$ \\
$\mathrm{C} 30-\mathrm{H} 30 \cdots \mathrm{O} 2^{\mathrm{v}}$ & 1.00 & 2.46 & $3.442(9)$ & $166(7)$
\end{tabular}

Symmetry codes: (i) $-x+1, y+1 / 2,-z+1$; (ii) $x, y, z+1$; (iii) $x-1, y, z-1$; (iv) $x, y, z-1$; (v) $x+1, y, z$. 